\title{
A mixed three-field FE formulation for stress accurate analysis including the incompressible limit
}

\author{
M. Chiumenti, M. Cervera and R. Codina \\ International Center for Numerical Methods in Engineering (CIMNE) \\ Universidad Politécnica de Cataluña (UPC) \\ Edificio C1, Campus Norte, Gran Capitán s/n, 08034 Barcelona Spain. \\ e-mail: michele@cimne.upc.edu,web page: http://www.cimne.com
}

KEYWORDS: Stress accurate, incompressible limit, Mixed three-field finite element technology, Variational Multi Scale (VMS) stabilization.

\begin{abstract}
In previous works, the authors have presented the stabilized mixed displacement/pressure formulation to deal with the incompressibility constraint. More recently, the authors have derived stable mixed stress/displ-acement formulations using linear/linear interpolations to enhance stress accuracy in both linear and non-linear problems. In both cases, the Variational Multi Scale (VMS) stabilization technique and, in particular, the Orthogonal Subgrid Scale (OSS) method allows the use of linear/linear interpolations for triangular and tetrahedral elements bypassing the strictness of the Inf-Sup condition on the choice of the interpolation spaces. These stabilization procedures lead to discrete problems which are fully stable, free of volumetric locking or stress oscillations.

This work exploits the concept of mixed finite element methods to formulate stable displacement/stress/pressure finite elements aimed for the solution of nonlinear problems for both solid and fluid finite element (FE) analyses. The final goal is to design a finite element technology able to tackle simultaneously problems which may involve isochoric behaviour (preserve the original volume) of the strain field together with high degree of accuracy of the stress field. These two features are crucial in nonlinear solid and fluid mechanics, as used in most numerical simulations of industrial manufacturing processes.

Numerical benchmarks show that the results obtained compare very favourably with those obtained with the corresponding mixed displacement/pressure formulation.
\end{abstract}




\section{Introduction}

This work presents a novel finite element technology with enhanced stress accuracy and, at the same time, able to deal with the fully incompressible behavior. Stress accuracy and performance in the incompressible limit are two requirements which often coexist when addressing the numerical simulation of different industrial manufacturing processes such as metal forming, forging, extrusion, friction stir welding, cutting or machining operations, among many others. The accuracy of the solution obtained by the numerical simulation of such industrial processes is directly related to the ability of the finite element technology adopted to deal with complex phenomena such as strain localization, the formation of shear bands, the prediction of crack propagation or the isochoric behavior of the inelastic (plastic) strains (fully deviatoric response of metallic materials under large deformations).

In the literature, the incompressible limit case and stress accuracy onhancement are generally treated separately. The problem posed by the incompressibility is to avoid the so called volumetric locking, an undesirable effect exhibited by finite element approximations based on the standard Galerkin approach. Successful strategies to avoid volumetric locking based on mixed formulations ([29], [2]), enhanced assumed strain methods ([33], [34], [7], [32], [28]) and nodal pressure and strain averaging ([24], [8], [9], [10], [35]) can be found in the literature.

Techniques based on the Variational Multi Scale (VMS) approach proposed by Hughes [26] have been applied in the context of solid mechanics in strain localization problems (see [27]). More

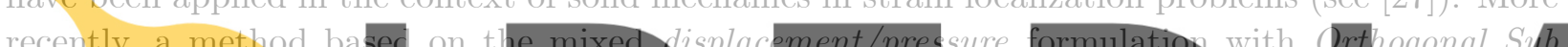

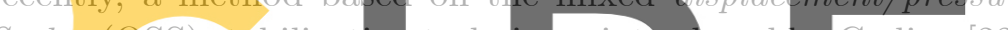
Scales (OSS) stabilizat elasticity by the authos

technique encouraged [1]) and to strain locali: ([14], [15] and [16]).
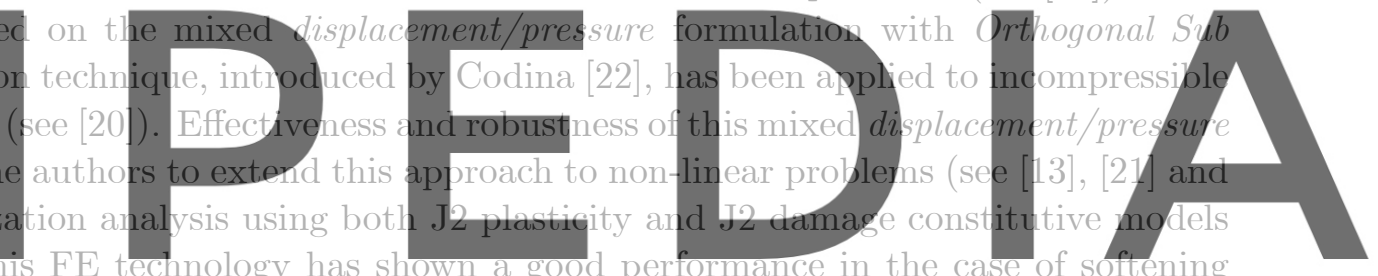

behavior of the material once the elastic limit is reached. In this case, strains concentrate into

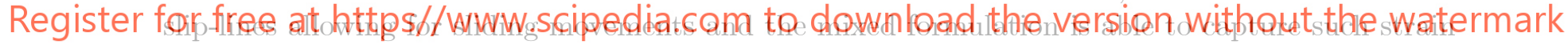

localization with practically mesh independent solutions.

However, even if the incompressible limit has been successfully tackled, the accuracy of the stress field approximation was still an open issue solved by local mesh refinement (see [31]).

Lately, the authors have proposed a mixed stress/displacement formulation which uses linear/linear interpolations for both master fields. Also in this case, the strictness of the inf-sup condition [11], when the standard Galerkin method is applied to mixed elements, imposes severe restrictions on the compatibility of the interpolations used for the displacement and the stress fields (see [30] and [3,4] for the analysis of admissible elements in linear elasticity). This difficulty can be circumvented again adopting a VMS approach which adds a consistent (reduced upon mesh refinement) residual-based stabilization to the original problem. This mixed stress/displacement finite element technology has demonstrated enhanced stress accuracy in both linear and non-linear analysis as well as the ability to capture stress concentrations and strain localizations with the guarantee of stress convergence upon mesh refinement. This is an essential requirement which cannot be accomplished in a point-wise manner using the standard Galerkin displacement-based formulation. An accurate estimation of the stress field even in the strain localization zone drives the crack propagation without the help of ad-hoc tracking algorithms. 
The present work makes a step forward introducing a mixed three-field formulation based on displacement/stress/pressure elements with linear (or, in general, equal) interpolations for all master fields. The only requirement is the split of the constitutive equation into volumetric and deviatoric parts (more details in Section 3). Once more, the stabilization technique to overcome the Inf-sup condition is presented in terms of the VMS method. The different assumptions and approximations used to derive this novel finite element technology are proposed in a very general format, applicable to $2 \mathrm{D}$ and $3 \mathrm{D}$ problems. Section 4 deals with the implementation and computational aspects. Finally, Section 5 shows the performance of the proposed formulation comparing with the well established mixed displacement-pressure formulation.

\section{The continuum problem statement}

Let us denote by $\Omega$ an open and bounded domain in $\mathbb{R}^{n_{\text {dim }}}$ where $n_{\text {dim }}$ is the number of dimensions of the space, and $\partial \Omega$ its boundary. The boundary $\partial \Omega$ is split into $\partial \Omega_{u}$ and $\partial \Omega_{t}$, being $\partial \Omega=\partial \Omega_{u} \cup \partial \Omega_{t}$ such that the prescribed displacements, $\overline{\mathbf{u}}$, are specified on $\partial \Omega_{u}$ (Dirichlet boundary conditions) and the prescribed tractions, $\overline{\mathrm{t}}$, are applied on $\partial \Omega_{t}$ (Neumann boundary conditions).

The continuum mechanical problem of linear elasticity to be considered is defined by the following system of equations:

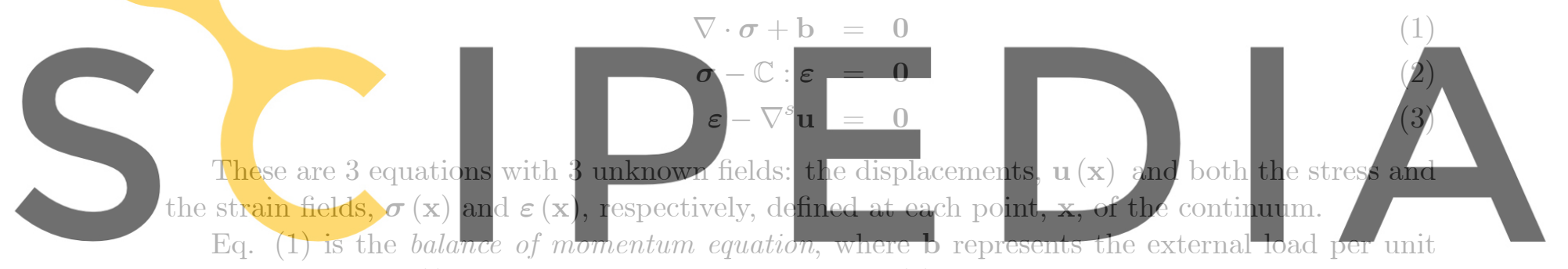

of volume and $\nabla \cdot(\cdot)$ is the divergence operator. Eq. (2) is the constitutive equation for linear

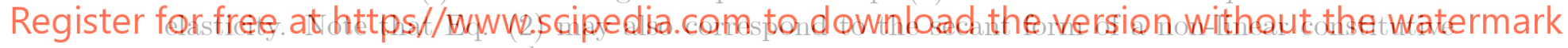

equation, where $\mathbb{C}$ is the $4^{\text {th }}$ order (secant) constitutive tensor. Finally, Eq. (3) is the kinematic equation (in the hypothesis of infinitesimal strains), where $\nabla^{s}(\cdot)=\frac{1}{2}\left[\nabla(\cdot)+\nabla(\cdot)^{T}\right]$ denotes the symmetric gradient operator and $\nabla(\cdot)$ is the gradient operator.

There are different alternatives to solve problem $(1-3)$ with the corresponding appropriate boundary conditions described.

The classical displacement-based formulation is obtained by substituting Eqs. (2) and (3) into Eq. (1). The result is Navier's equation

$$
\nabla \cdot\left(\mathbb{C}: \nabla^{s} \mathbf{u}\right)+\mathbf{b}=\mathbf{0}
$$

which is written in terms of the displacement field only.

Alternatively, the mixed $\mathbf{u} / \boldsymbol{\sigma}$ formulation uses both stresses and displacements as master fields as:

$$
\begin{array}{r}
\nabla \cdot \boldsymbol{\sigma}+\mathbf{b}=\mathbf{0} \\
\boldsymbol{\sigma}-\mathbb{C}: \nabla^{s} \mathbf{u}=\mathbf{0}
\end{array}
$$

obtained by substituting Eq.(3) into Eq.(2). 


\section{The volumetric/deviatoric split}

The objective of this section is the split of the constitutive equation (2) into its volumetric and deviatoric parts. This is possible in the cases of linear elasticity (compressible or incompressible), J2-plasticity (both small and large strain hypotheses), isotropic damage, Newtonian and non-Newtonian flows as Norton-Hoff, Sheppard-Wright, Bingham visco-plastic flow, among others. The volumetric/deviatoric split is the starting point to develop a formulation able to tackle the incompressible limit.

\subsection{Volumetric and deviatoric operators}

Let us define the $4^{\text {th }}$ rank volumetric and deviatoric tensors, $\mathbb{V}$ and $\mathbb{P}$, as follows:

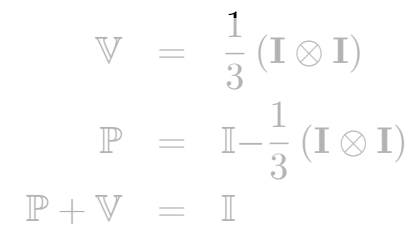

where $\mathbb{I}=\left[\delta_{i j} \delta_{k l}\right]$ and $\mathbf{I}=\left[\delta_{i j}\right]$ are the $4^{\text {th }}$ rank and the $2^{\text {nd }}$ rank identity tensors, respectively $\left(\delta_{i j}\right.$ is Kronecker's delta). $\mathbb{V}$ and $\mathbb{P}$ can be also thought as operators acting on second order tensors by

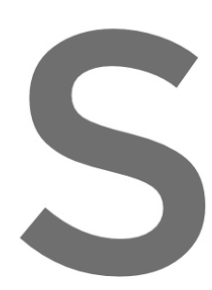
taking double contract
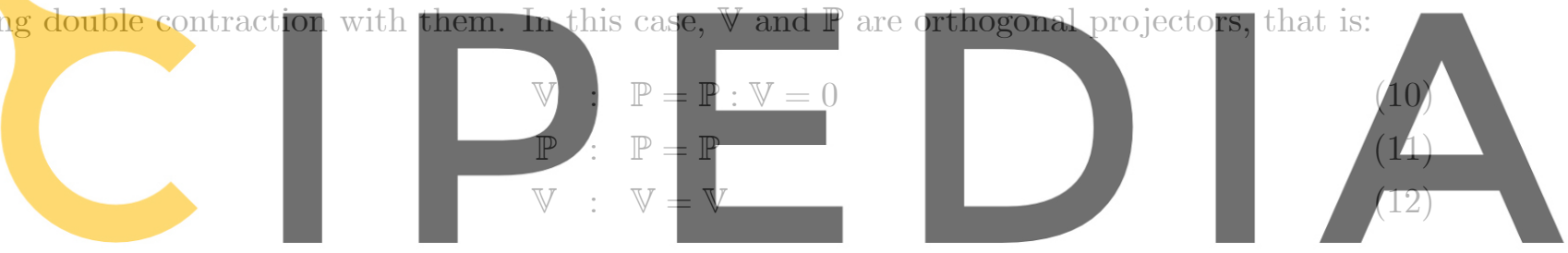

\subsection{Split of stress and strain tensors}

Register for free at https//www.scipedia.com to download the version without the watermark

Using $\mathbb{V}$ and $\mathbb{P}$ operators, it is possible to extract the spheric and the deviatoric parts of a generic

$2^{\text {nd }}$ order tensor. Particularly, when applied to the stress tensor, $\sigma$, the result is:

$$
\begin{aligned}
& \mathbb{V}: \boldsymbol{\sigma}=\frac{1}{3}(\mathbf{I} \otimes \mathbf{I}): \boldsymbol{\sigma}=p \mathbf{I} \\
& \mathbb{P}: \boldsymbol{\sigma}=\left[\mathbb{I}-\frac{1}{3}(\mathbf{I} \otimes \mathbf{I})\right]: \boldsymbol{\sigma}=\boldsymbol{\sigma}-p \mathbf{I}=\mathbf{s}
\end{aligned}
$$

where $p(\boldsymbol{\sigma})=\frac{1}{3}(\boldsymbol{\sigma}: \mathbf{I})=\frac{1}{3} \operatorname{tr}(\boldsymbol{\sigma})$ is the pressure and $\mathbf{s}(\boldsymbol{\sigma})=\mathbb{P}: \boldsymbol{\sigma}=\operatorname{dev}(\boldsymbol{\sigma})$ are the deviatoric stresses. On one hand, the stress tensor, $\sigma$, is symmetric and consists of 6 independent components. On the other hand, the deviatoric stress tensor, s, is also defined by 6 components but only 5 of them are independent, as the deviatoric stresses must respect the constraint: $\operatorname{tr}(\mathbf{s})=0$. This poses the question of how to select a frame independent (not unique) basis for the deviatoric stress tensor.

This given, the stress tensor can be rebuilt adding both components of the split as:

$$
\boldsymbol{\sigma}=p \mathbf{I}+\mathbf{s}
$$

In a similar way, it is possible to split the strain tensor, $\varepsilon$, as: 


$$
\begin{aligned}
& \mathbb{V}: \varepsilon=\frac{1}{3}(\mathbf{I} \otimes \mathbf{I}): \varepsilon=\frac{1}{3} e_{v o l} \mathbf{I} \\
& \mathbb{P}: \boldsymbol{\varepsilon}=\left[\mathbb{I}-\frac{\mathbf{1}}{\mathbf{3}}(\mathbf{I} \otimes \mathbf{I})\right]: \varepsilon=\varepsilon-\frac{1}{3} e_{v o l} \mathbf{I}=\mathbf{e}
\end{aligned}
$$

where $e_{v o l}=\operatorname{tr}(\varepsilon)$ is the volumetric deformation and $\mathbf{e}=\operatorname{dev}(\varepsilon)$ accounts for the distortions. The resulting split format of the strain tensor is:

$$
\varepsilon=\frac{1}{3} e_{v o l} \mathbf{I}+\mathbf{e}
$$

\subsection{Split of the kinematic equation}

Within the hypothesis of infinitesimal strains the kinematic equation is expressed as:

$$
\varepsilon=\nabla^{s} \mathbf{u}
$$

Applying the volumetric/deviatoric operators, equation (19) is split as follows:
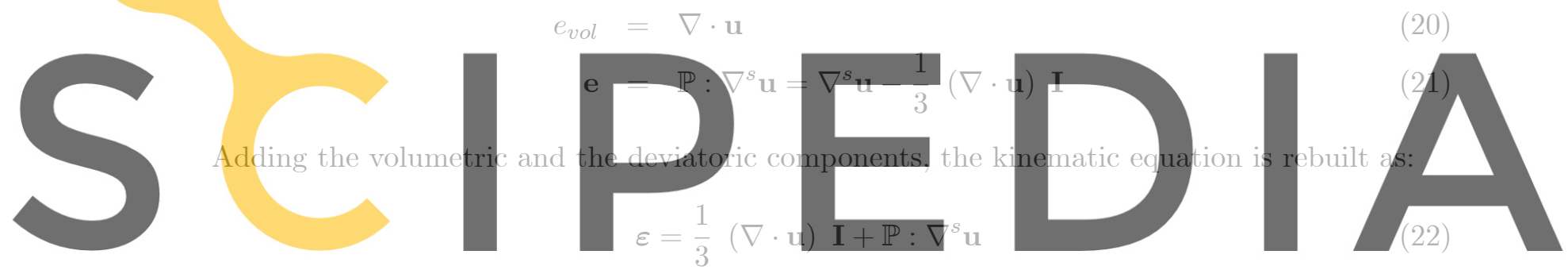

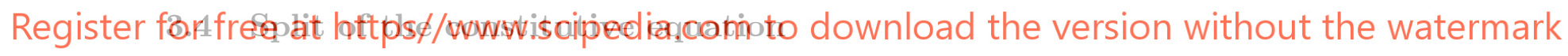

Let us assume that the constitutive relationship between stresses and strains can be expressed, in secant form, as:

$$
\sigma=\mathbb{C}: \varepsilon
$$

Then the spheric and the deviatoric parts of the constitutive tensor, $\mathbb{C}^{v o l}$ and $\mathbb{C}^{\text {dev }}$, respectively, are obtained as:

$$
\begin{aligned}
\mathbb{C}^{\text {vol }} & =\mathbb{V}: \mathbb{C} \\
\mathbb{C}^{\text {dev }} & =\mathbb{P}: \mathbb{C} \\
\mathbb{C} & =\mathbb{C}^{\text {vol }}+\mathbb{C}^{\text {dev }}
\end{aligned}
$$

Introducing the split of stresses and strains ((15) and (18), respectively), the constitutive relationship in (23) can be written as:

$$
(p \mathbf{I}+\mathbf{s})=\left(\mathbb{C}^{v o l}+\mathbb{C}^{d e v}\right):\left(\frac{1}{3} e_{v o l} \mathbf{I}+\mathbf{e}\right)
$$


that is:

$$
\begin{aligned}
& p=C^{v o l} e_{v o l} \\
& \mathbf{s}=\mathbb{C}^{d e v}: \mathbf{e}
\end{aligned}
$$

which are the volumetric and the deviatoric counterparts of the original constitutive equation, being $C^{v o l}=\frac{1}{9} \mathbf{I}: \mathbb{C}^{v o l}: \mathbf{I}$ the bulk modulus of the material.

Particularizing to linear isotropic elasticity, the constitutive tensor is given by:

$$
\mathbb{C}=K(\mathbf{I} \otimes \mathbf{I})+2 G\left[\mathbb{I}-\frac{1}{3}(\mathbf{I} \otimes \mathbf{I})\right]
$$

where $K$ is the (elastic) bulk modulus and $G$ is the (elastic) shear modulus. Therefore, the spheric and the deviatoric parts of the elastic constitutive tensor (30) are:

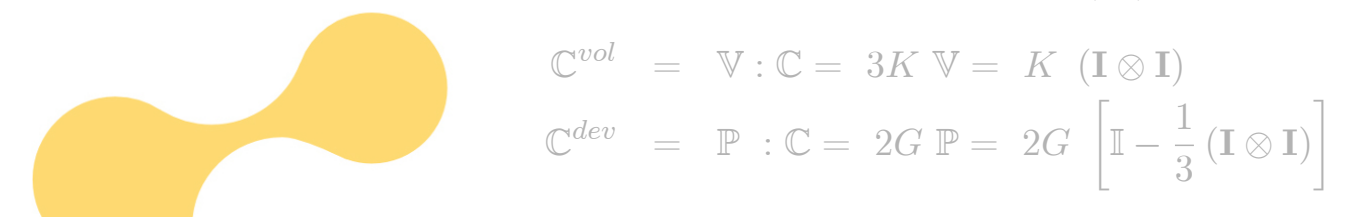

and $C^{v o l}=K$.

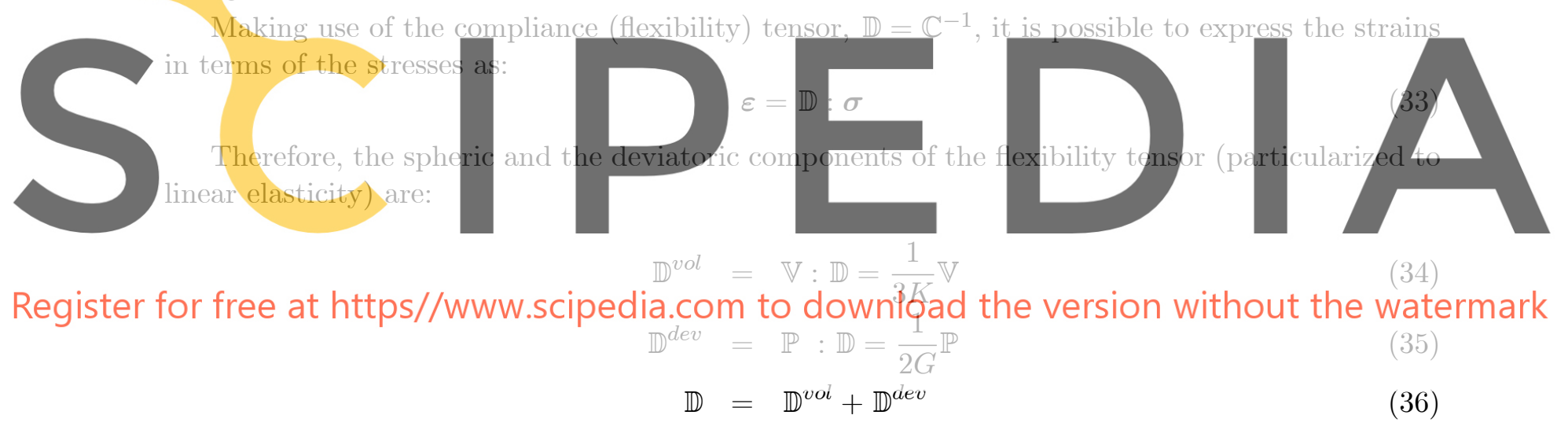

Introducing the split format of the stresses (Eq. (15)), the strains (Eq. (18)) and the split of the flexibility tensor (Eq. (36)) into Eq. (33), the result is:

$$
\left(\frac{1}{3} e_{v o l} \mathbf{I}+\mathbf{e}\right)=\left(\mathbb{D}^{v o l}+\mathbb{D}^{d e v}\right):(p \mathbf{I}+\mathbf{s})
$$

where it is possible to identify the volumetric and the deviatoric expressions of the original constitutive equation (33):

$$
\begin{aligned}
e_{v o l} & =D^{v o l} p \\
\mathbf{e} & =\mathbb{D}^{d e v}: \mathbf{s}
\end{aligned}
$$

being $D^{\text {vol }}=\mathbf{I}: \mathbb{D}^{\text {vol }}: \mathbf{I}$ the compressibility modulus. 
Finally, making use of the kinematic equations (20) and (21), the previous relations translate into:

$$
\begin{aligned}
\nabla \cdot \mathbf{u} & =D^{v o l} p \\
\mathbb{P}: \nabla^{s} \mathbf{u} & =\mathbb{D}^{d e v}: \mathbf{s}
\end{aligned}
$$

and, in the incompressible limit $D^{v o l} \rightarrow 0$, they reduce to:

$$
\begin{aligned}
\nabla \cdot \mathbf{u} & =0 \\
\mathbb{P}: \nabla^{s} \mathbf{u} & =\mathbb{D}^{d e v}: \mathbf{s}
\end{aligned}
$$

Observe that the constitutive relationship in the split format above consists of 6 equations (the same as for equation (27)). In fact (28) is a single equation while (29) develops into 5 equations.

The particularization to linear elasticity is:

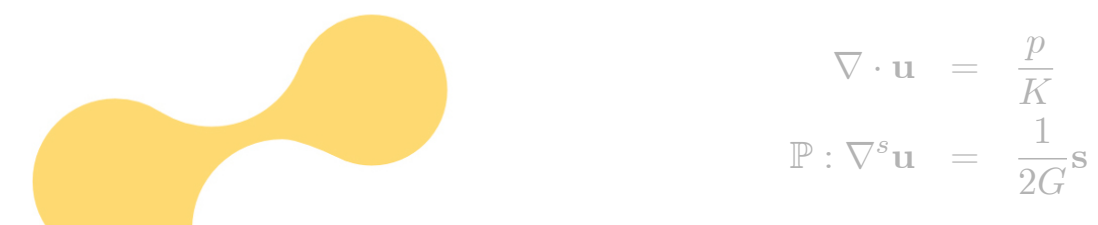

and, in the incompressible limit, $K \rightarrow \infty$, they reduce to:
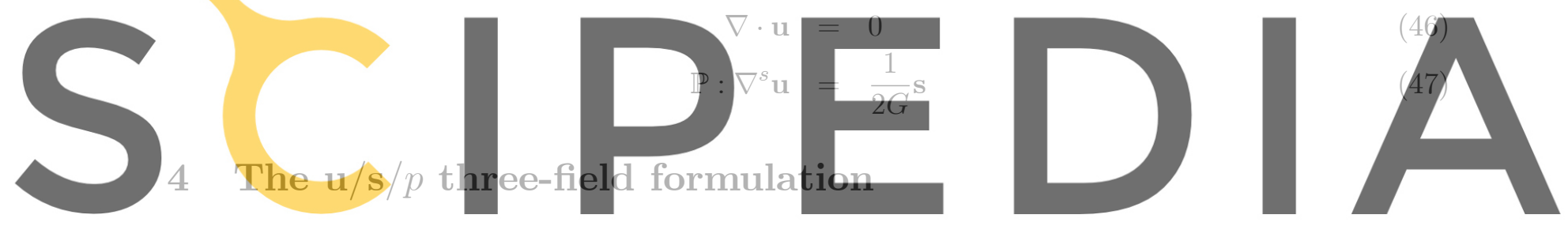

In this section, a novel three-field formulation is introduced. The objective is the definition of

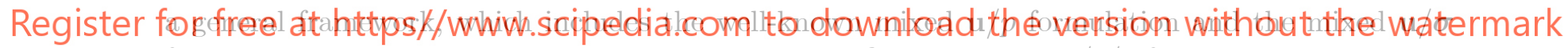

formulation as particular cases. To this end, let us define the mixed u/s/p formulation, which uses the displacement field, u, together with the deviatoric component of the stresses, s, and the pressure field, $p$, as independent variables. Hence, the governing equations of the problem are rewritten as:

$$
\begin{array}{r}
\nabla \cdot \mathbf{s}+\nabla p+\mathbf{b}=\mathbf{0} \\
\mathbb{P}: \nabla^{s} \mathbf{u}-\mathbb{D}^{d e v}: \mathbf{s}=\mathbf{0} \\
\nabla \cdot \mathbf{u}-D^{v o l} p=0
\end{array}
$$

where Eq.(48) is the balance of momentum equation in mixed form. The 5 equations in (49) together with the scalar equation in (50) are the deviatoric and the volumetric counterparts of a generic constitutive equation as presented in $(41-40)$.

The weak form of the mixed $\mathbf{u} / \mathbf{s} / p$ formulation reads:

$$
\begin{aligned}
(\mathbf{v}, \nabla \cdot \mathbf{s})+(\mathbf{v}, \nabla p)+(\mathbf{v}, \mathbf{b}) & =0 \text { in } \Omega \\
\left(\boldsymbol{\xi}, \mathbb{P}: \nabla^{s} \mathbf{u}\right)-\left(\boldsymbol{\xi}, \mathbb{D}^{d e v}: \mathbf{s}\right) & =0 \text { in } \Omega \\
(q, \nabla \cdot \mathbf{u})-D^{v o l}(q, p) & =0 \text { in } \Omega
\end{aligned}
$$


where $\mathbf{v}$ (vector), $q$ (scalar) and $\boldsymbol{\xi}$ (a tensorial field of 5 independent components) are the variations of the displacement, the pressure field and the deviatoric stresses, respectively, and $(\cdot, \cdot)_{D}$ denotes the integral of the product of two functions in a domain $D$, which is omitted when $D \equiv \Omega$. Integrating Eq.(51) by parts and taking $\mathbf{v}=\mathbf{0}$ on $\partial \Omega_{u}$, the problem can be written as:

$$
\begin{aligned}
& 0+\left(\nabla^{s} \mathbf{v}, \mathbf{s}\right)+(\nabla \cdot \mathbf{v}, p)=F(\mathbf{v}) \\
& \left(\boldsymbol{\xi}, \mathbb{P}: \nabla^{s} \mathbf{u}\right)-\left(\boldsymbol{\xi}, \mathbb{D}^{\text {dev }}: \mathbf{s}\right)+0 \quad=0 \\
& (q, \nabla \cdot \mathbf{u}) \quad+\quad 0 \quad-\quad D^{v o l}(q, p)=0
\end{aligned}
$$

where

$$
F(\mathbf{v})=(\mathbf{v}, \mathbf{b})+(\mathbf{v}, \overline{\mathbf{t}})_{\partial \Omega_{t}}
$$

is the work of the external loads.

Problem (54) involves the first derivatives of $\mathbf{u}(\mathbf{x})$. Hence, the natural space for the continuum displacements field is: $\mathbf{u}(\mathbf{x}) \in V=H^{1}(\Omega)^{n_{\mathrm{dim}}} \cdot H^{m}(\Omega)$ denotes the space of functions whose derivatives (up to order $m \geq 0$ ) belong to $L^{2}(\Omega)$. The corresponding variations are defined in: $\mathbf{v}(\mathbf{x}) \in V_{0}=\left\{\mathbf{v}(\mathbf{x}) \in V \mid \mathbf{v}=\mathbf{0}\right.$ for $\left.\forall \mathbf{x} \in \partial \Omega_{u}\right\}$.

The pressure field, $p$, and its variation, $q$, belong to space $Q=L^{2}(\Omega)$, while the natural space for deviatoric part of the stress field, $\mathbf{s}$, and its variations, $\boldsymbol{\xi}$, is $\widehat{S}=\left\{\mathrm{s}(\mathrm{x})=\left[s_{i j}(\mathrm{x})\right]\right.$,

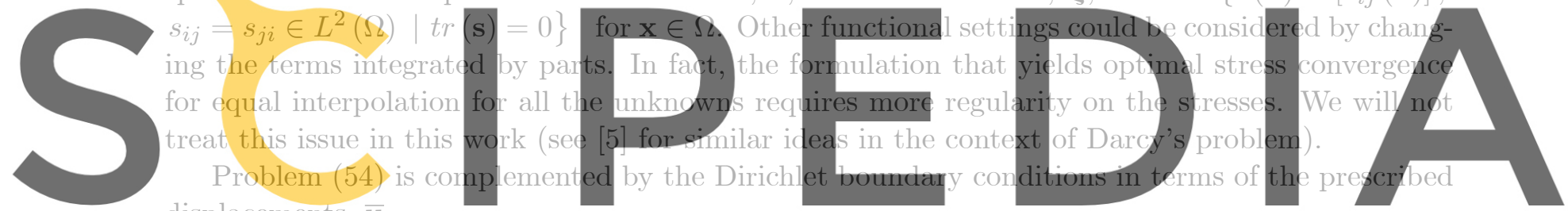

displacements, $\overline{\mathrm{u}}$.

Register for free at https//www.scipedia.com to download the version without the watermark

Remark 1 To achieve symmetry, it is possible to substitute the second term in the first equation,

$\left(\nabla^{s} \mathrm{v}, \mathrm{s}\right)$, by the equivalent term: $\left(\mathbb{P}: \nabla^{s} \mathrm{v}, \mathrm{s}\right)$. Then, problem (54) reads:

$$
\begin{array}{cccccc}
0 & +\left(\mathbb{P}: \nabla^{s} \mathbf{v}, \mathbf{s}\right) & +(\nabla \cdot \mathbf{v}, p) & = & F(\mathbf{v}) \\
\left(\boldsymbol{\xi}, \mathbb{P}: \nabla^{s} \mathbf{u}\right) & -\left(\boldsymbol{\xi}, \mathbb{D}^{d e v}: \mathbf{s}\right)+ & 0 & = \\
(q, \nabla \cdot \mathbf{u})+ & 0 & - & D^{v o l}(q, p) & = & 0
\end{array}
$$

Remark 2 Alternatively, the second equation in (56) can be tested using the test functions, $\gamma=$ $q \mathbf{I}+\boldsymbol{\xi}$ with $q \in Q$ and $\boldsymbol{\xi} \in \widehat{S}$, in the form:

$$
\left(\boldsymbol{\gamma}, \mathbb{P}: \nabla^{s} \mathbf{u}\right)-\left(\gamma, \mathbb{D}^{d e v}: \mathbf{s}\right)=0
$$

This equation is equivalent to the original one because the extra terms, $\left(q \mathbf{I}, \mathbb{P}: \nabla^{s} \mathbf{u}\right)$ and $\left(q \mathbf{I}, \mathbb{D}^{\text {dev }}: \mathbf{s}\right)$, are null by construction. However, Eq.(57) develops into 6 scalar equations being only 5 of them linearly independent. Eq.(57) admits as a solution a stress field, $\hat{\mathbf{s}}$, where $\operatorname{tr}(\hat{\mathbf{s}}) \neq 0$ is not defined. 
To overcome this inconvenient, it is necessary to prescribe the volumetric part of the stress field $\hat{\mathbf{s}}$. One possibility is to add the term $\left(\gamma, \mathbb{D}^{v o l}: \hat{\mathbf{s}}\right)$ to Eq. (57), which corresponds to the weak form of the constraint: $\operatorname{tr}(\hat{\mathbf{s}})=0$. The result is the following equation:

$$
\left(\gamma, \mathbb{P}: \nabla^{s} \mathbf{u}\right)-(\gamma, \mathbb{D}: \hat{\mathbf{s}})=0
$$

and problem (56) can be reformulated as:

$$
\begin{array}{cccccc}
0 & +\left(\mathbb{P}: \nabla^{s} \mathbf{v}, \hat{\mathbf{s}}\right) & + & (\nabla \cdot \mathbf{v}, p) & = & F(\mathbf{v}) \\
\left(\boldsymbol{\gamma}, \mathbb{P}: \nabla^{s} \mathbf{u}\right) & - & (\boldsymbol{\gamma}, \mathbb{D}: \hat{\mathbf{s}}) & + & 0 & = \\
(q, \nabla \cdot \mathbf{u})+ & 0 & - & D^{\text {vol }}(q, p) & = & 0
\end{array}
$$

where the stress field, $\hat{\mathrm{s}}$, is deviatoric in a weak sense only.

\section{Discrete approximation for the three-field formulation}

\subsection{Galerkin approach}

Let us now define the discrete Galerkin finite element counterpart of problem (56) as:
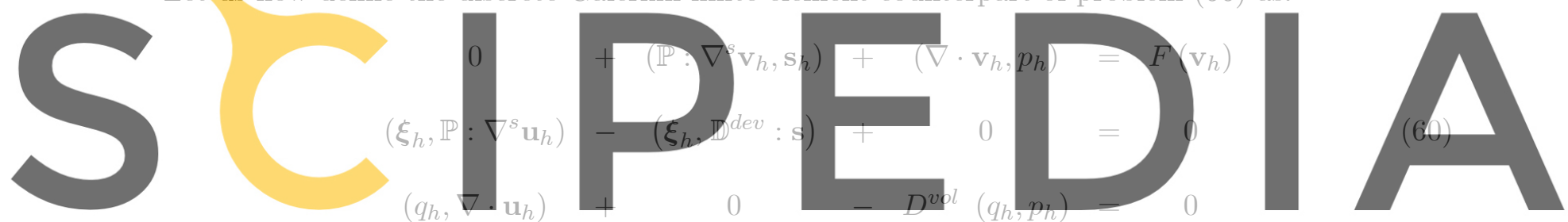

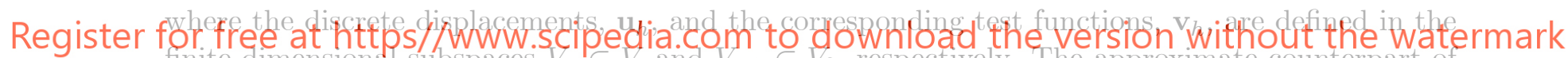
finite-dimensional subspaces $V_{h} \subset V_{\text {and }} V_{0, h} \subset V_{0}$, respectively. The approximate counterpart of

the stress field, $\mathrm{s}_{h}$ and the pressure field, $p_{h}$, together with their variations, $\xi_{h}$ and $q_{h}$, belong to the finite element spaces $S_{h} \subset S$ and $Q_{h} \subset Q$, respectively. Standard conforming approximations are considered.

In the following, we will be interested in continuous finite element spaces $V_{h}, \widehat{S}_{h}$ and $Q_{h}$ and, more specifically, in equal interpolation for displacements, stresses and pressures.

From the computational point of view, it is interesting to adopt Voigt's notation, which transforms the tensorial format of a generic symmetric tensor into a 6 -dimensional vector. In the Cartesian system, the stress and strain tensors are expressed using Voigt's notation as:

$$
\begin{aligned}
& \mathbf{s}=\left[\begin{array}{llllll}
s_{x x} & s_{y y} & s_{z z} & s_{x y} & s_{x z} & s_{y z}
\end{array}\right]=\left[s_{i}\right] \\
& \boldsymbol{\varepsilon}=\left[\begin{array}{llllll}
\varepsilon_{x x} & \varepsilon_{y y} & \varepsilon_{z z} & \gamma_{x y} & \gamma_{x z} & \gamma_{y z}
\end{array}\right]=\left[\varepsilon_{i}\right]
\end{aligned}
$$

where $\gamma_{i j}=2 \varepsilon_{i j}$ are the so-called engineering strains.

Let $n_{n o d}$ be the number of nodes per element of the finite element partition. Then, $\mathbf{U}_{h}=\left[U_{i}^{A}\right]$ is an array of dimension $3 n_{n o d}$ with 3 components (lower subindex, $i$ ) of displacements at each node (upper subindex $A$ ) of the finite element. Similarly, $\mathbf{S}_{h}=\left[S_{i}^{A}\right]$ is an array of dimension $6 n_{\text {nod }}$ 
with the 6 components of the deviatoric stresses per node. Finally, $\mathbf{P}_{h}=\left[P^{A}\right]$ is the array with the nodal values of the pressure field. The approximations of the master fields within each element result in:

$$
\begin{aligned}
\mathbf{u}_{h} & =\mathbf{N}_{u} \mathbf{U}_{h}=\left[N^{A} I_{(3 \times 3) i j} U_{j}^{A}\right] \\
\mathbf{s}_{h} & =\mathbf{N}_{s} \mathbf{S}_{h}=\left[N^{A} I_{(6 \times 6) i j} S_{j}^{A}\right] \\
p_{h} & =\mathbf{N}_{p} \mathbf{P}_{h}=\left[N^{A} P^{A}\right]
\end{aligned}
$$

where, denoting by $N^{A}$ the shape functions of the selected element type used in the FE partition, $\mathbf{N}_{u}$ is a $3 \times 3 n_{\text {nod }}$ matrix made of $n_{\text {nod }}$ blocks of the form $N^{A} \mathbf{I}_{(3 \times 3)}$. Similarly, $\mathbf{N}_{s}$ is a $6 \times 6 n_{\text {nod }}$ matrix made of $n_{\text {nod }}$ blocks of the form $N^{A} \mathbf{I}_{(6 \times 6)}$ and $\mathbf{N}_{p}=\left[N^{A}\right]$, with $A$ running from 1 to $n_{\text {nod }}$.

The components of the deviatoric stress tensor $\mathbf{S}_{h}$ are not independent, as the trace of the tensor is null. This condition can be imposed through a linear dependency matrix, $\mathbb{H}=\left[H_{i j}^{A B}\right]$, $\left(6 n_{\text {nod }} \times 5 n_{\text {nod }}\right)$, so that $\mathbf{S}_{h}=\mathbf{H} \widehat{\mathbf{S}}_{h}$. This given, $\widehat{\mathbf{S}}_{h}=\left[\widehat{S}_{i}^{A}\right]$ results in an array of dimension $5 n_{\text {nod }}$ with 5 independent components of the deviatoric stress tensor at each node of the finite element:

$$
\mathbf{s}_{h}=\mathbf{N}_{s} \mathbf{S}_{h}=\mathbf{N}_{s}\left(\mathbf{H} \widehat{\mathbf{S}}_{h}\right)=\left(\mathbf{N}_{s} \mathbf{H}\right) \widehat{\mathbf{S}}_{h}=\widehat{\mathbf{N}}_{s} \widehat{\mathbf{S}}_{h}
$$

where $\widehat{\mathbf{N}}_{s}=\mathbf{N}_{s} \mathbf{H}$.

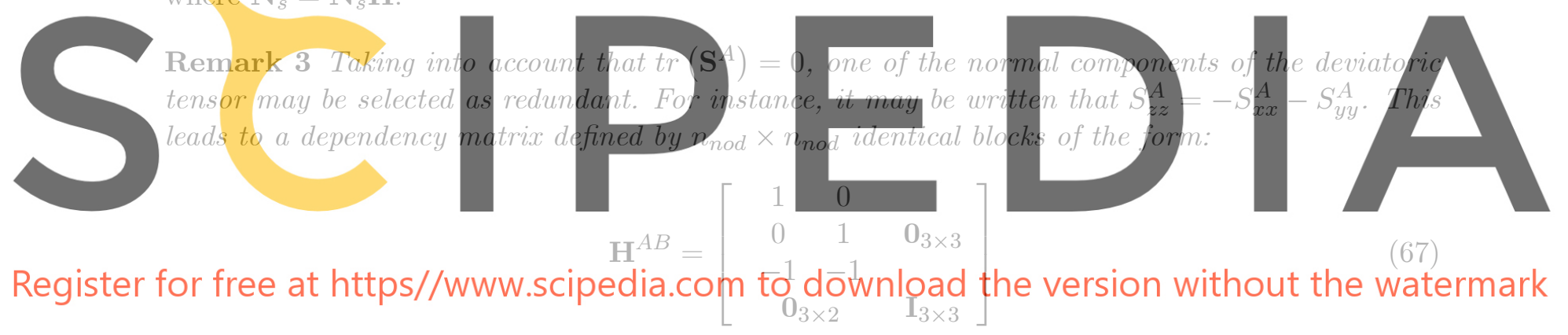

Note that the selection of the redundant normal deviatoric stress component is arbitrary.

The following arrays are also introduced (for the $3 D$ case):

$$
\begin{aligned}
\mathbf{G}^{A}= & {\left[\begin{array}{lll}
\frac{\partial N^{A}}{\partial X} & \frac{\partial N^{A}}{\partial Y} & \frac{\partial N^{A}}{\partial Z}
\end{array}\right]^{T} } \\
\mathbf{B}^{A}= & {\left[\begin{array}{cccccc}
\frac{\partial N^{A}}{\partial X} & 0 & 0 & \frac{\partial N^{A}}{\partial Y} & \frac{\partial N^{A}}{\partial Z} & 0 \\
0 & \frac{\partial N^{A}}{\partial Y} & 0 & \frac{\partial N^{A}}{\partial X} & 0 & \frac{\partial N^{A}}{\partial Z} \\
0 & 0 & \frac{\partial N^{A}}{\partial Z} & 0 & \frac{\partial N^{A}}{\partial X} & \frac{\partial N^{A}}{\partial Y}
\end{array}\right]^{T} }
\end{aligned}
$$

On one side, $\mathbf{G}=\left[G_{i}^{A}\right]$ and $\mathbf{G}^{T}$ are the (discrete) gradient and divergence operators, respectively. On the other side, $\mathbf{B}=\left[B_{i j}^{A}\right]$ and $\mathbf{B}^{T}$ stand for the (discrete) symmetric gradient and divergence (of a tensor field expressed in Voigt's notation) operators, respectively.

The elemental stiffness matrix (upper subindex $(e)$ denotes a generic element of the FE discretization) of the algebraic form of problem (60) is: 


$$
\mathbb{K}_{h}^{(e)}=\int_{\Omega^{(e)}}\left[\begin{array}{ccc}
{[\mathbf{0}]} & {\left[\mathbf{B}^{T} \mathbf{P} \widehat{\mathbf{N}}_{s}\right]} & {\left[\mathbf{G N}_{p}\right]} \\
{\left[\widehat{\mathbf{N}}_{s}^{T} \mathbf{P B}\right]} & -\left[\widehat{\mathbf{N}}_{s}^{T} \mathbf{D}^{d e v} \widehat{\mathbf{N}}_{s}\right] & {[\mathbf{0}]} \\
{\left[\mathbf{N}_{p}^{T} \mathbf{G}^{T}\right]} & {[\mathbf{0}]} & -D^{v o l}\left[\mathbf{N}_{p}^{T} \mathbf{N}_{p}\right]
\end{array}\right] d \Omega
$$

where according to the Galerkin method, the same span of interpolation (shape) functions are used as weight functions.

The deviatoric operator $\mathbb{P}$ has been replaced by the $6 \times 6$ matrix $\mathbf{P}=\mathbf{I}-\frac{1}{3}(\mathbf{1} \otimes \mathbf{1})$, that is an operator of rank two used to extract the deviatoric component of a symmetric tensor (expressed in Voigt's notation), being $\mathbf{1}=\left[\begin{array}{llllll}1 & 1 & 1 & 0 & 0 & 0\end{array}\right]$.

Remark 4 The elemental stiffness matrix coming from the discrete counterpart of the alternative format of the three-field problem (59) is:

$$
\mathbb{K}_{h}^{(e)}=\int_{\Omega^{(e)}}\left[\begin{array}{ccc}
{[\mathbf{0}]} & {\left[\mathbf{B}^{T} \mathbf{P} \mathbf{N}_{s}\right]} & {\left[\mathbf{G} \mathbf{N}_{p}\right]} \\
{\left[\mathbf{N}_{s}^{T} \mathbf{P B}\right]} & -\left[\mathbf{N}_{s}^{T} \mathbf{D} \mathbf{N}_{s}\right] & {[\mathbf{0}]} \\
{\left[\mathbf{N}_{p}^{T} \mathbf{G}^{T}\right]} & {[\mathbf{0}]} & -D^{v o l}\left[\mathbf{N}_{p}^{T} \mathbf{N}_{p}\right]
\end{array}\right] d \Omega
$$

where now the nodal stresses are $\mathbf{S}_{h}=\left[S_{i}^{A}\right]$ with the corresponding interpolation functions $\mathbf{N}_{s}$. Observe that the stress tensor at each node of the element, $\mathbf{S}^{A}$, is deviatoric in weak sense only.

\subsection{Variational-Multi-Scale Stabilization technique}

The stability of the discrete formulation depends on compatibility restrictions on the interpolation functions chosen for the displacement, stress and pressure fields, as stated by the appropriate inf-sup condition (see [11]). According to these restrictions, mixed elements with continuous equal order linear interpolation for all fields are not stable. However, the inf-sup condition can be circumvented by using a stabilization technique.

In this work, the Variational-Multi-Scale (VMS) method is introduced to stabilize the discrete formulation of the mixed problem allowing for the use of linear interpolations for all master fields.

The basic idea of the VMS approach is to enlarge Galerkin's space of approximation, $\mathbb{W}_{h}=$ $V_{h} \times S_{h} \times Q_{h}$, adding a finer resolution space, $\widetilde{\mathbb{W}}=\widetilde{V}_{0} \times \widetilde{S} \times \widetilde{Q}$, referred to as the sub-grid scale. Subindex $(\cdot)_{0}$ means that the sub-grid scale defined for the displacement field, (defined at element level) vanishes at all the element boundaries.

Therefore, the enhanced approximation space is defined as:

$$
\mathbb{W} \simeq \mathbb{W}_{h} \oplus \widetilde{\mathbb{W}} \rightarrow\left\{\begin{array}{c}
\mathbf{u} \simeq \mathbf{u}_{h}+\widetilde{\mathbf{u}} \\
\mathbf{s} \simeq \mathbf{s}_{h}+\widetilde{\mathbf{s}} \\
p \simeq p_{h}+\widetilde{p}
\end{array}\right.
$$

where $\mathbb{W}_{h}$ and $\widetilde{\mathbb{W}}$ represent two different levels of resolution: one coarse and a finer one.

In order to ensure consistency of the stabilized mixed formulation, so that the discrete solution converges to the continuous solution on mesh refinement, the sub-grid scale is expressed in terms 
of the residual of the projected (Galerkin) counterpart of Eqs.(48 - 50), (see [23] for motivation)

$$
\begin{aligned}
& \widetilde{\mathbf{u}}=\widetilde{\boldsymbol{\tau}}_{u} R_{u}^{h}=\widetilde{\boldsymbol{\tau}}_{u}\left[\nabla \cdot \mathbf{s}_{h}+\nabla p_{h}+\mathbf{b}\right] \\
& \widetilde{\mathbf{s}}=\widetilde{\boldsymbol{\tau}}_{s} R_{s}^{h}=\widetilde{\boldsymbol{\tau}}_{s}:\left[\mathbb{P}: \nabla^{s} \mathbf{u}_{h}-\mathbb{D}^{d e v}: \mathbf{s}_{h}\right] \\
& \widetilde{p}=\widetilde{\tau}_{p} R_{p}^{h}=\widetilde{\tau}_{p}\left[\nabla \cdot \mathbf{u}_{h}-D^{v o l} p_{h}\right]
\end{aligned}
$$

In the incompressible limit, $D^{v o l} \rightarrow 0$ so that $R_{p}^{h}=\nabla \cdot \mathbf{u}_{h}$ and the pressure subscale reduces to:

$$
\widetilde{p}=\widetilde{\tau}_{p} R_{p}^{h}=\widetilde{\tau}_{p}\left[\nabla \cdot \mathbf{u}_{h}\right]
$$

The stabilization parameters:

$$
\begin{aligned}
& \widetilde{\boldsymbol{\tau}}_{u}=\tau_{u} \mathbf{I}_{3 \times 3} \quad \leftarrow \tau_{u}=c_{u} \frac{L h}{2 \widehat{G}} \\
& \widetilde{\boldsymbol{\tau}}_{s}=\tau_{s} \mathbb{C}^{d e v} \quad \leftarrow \quad \tau_{s}=c_{s} \frac{h}{L} \\
& \widetilde{\tau}_{p}=\tau_{p} \widetilde{C}^{v o l} \quad \leftarrow \quad \tau_{p}=c_{p} \frac{h}{L}
\end{aligned}
$$

are expressed in terms of a characteristic length of the problem, $L$, the element size, $h$, and the secant shear modulus $2 \widehat{G}=\left\|\mathbf{s}_{h}\right\| /\left\|\mathbf{e}_{h}\right\|$. Coefficient $c_{u}, c_{s}$ and $c_{p}$ are constants to be chosen. The definition of the stabilization parameters (77) is optimal when equal interpolation is adopted for all the master fields (see [5] and the discussion in [17] for the mixed $\mathbf{u} / \boldsymbol{\sigma}$ approach).

$\widetilde{C}^{v o l}=\min \left(K, \frac{2}{3} G\right)$ is the compressible parameter (only) used for the stabilization: for the elastic problem $\widetilde{C}^{\text {vol }}=C^{\text {vol }}=K$ (compressible analysis, $K \leqq \frac{2}{3} G$ ) and $\widetilde{C}^{\text {vol }}=\frac{2}{3} G$ (incompressible limit, $K>\frac{2}{3} G$ ).

This given, the solution of the compressible problem is approximated as:

$$
\begin{aligned}
& \mathbf{u} \simeq \mathbf{u}_{h}+\widetilde{\mathbf{u}}=\mathbf{u}_{h}+\tau_{u}\left[\nabla \cdot \mathbf{s}_{h}+\nabla p_{h}+\mathbf{b}\right] \\
& \mathbf{s} \simeq \mathbf{s}_{h}+\widetilde{\mathbf{s}}=\left(1-\tau_{s}\right) \mathbf{s}_{h}+\tau_{s}\left[\mathbb{C}^{d e v}: \nabla^{s} \mathbf{u}_{h}\right] \\
& p \simeq p_{h}+\widetilde{p}=\left(1-\tau_{p}\right) p_{h}+\tau_{p}\left[C^{v o l} \nabla \cdot \mathbf{u}_{h}\right]
\end{aligned}
$$

while in the incompressible limit, equation (80) reduces to:

$$
p \simeq p_{h}+\widetilde{p}=p_{h}+\tau_{p}\left[\widetilde{C}^{v o l} \nabla \cdot \mathbf{u}_{h}\right]
$$

Remark 5 The sub-grid scale contributions are discontinuous but, even if they are computed element-wise, they cannot be condensed at element level, because $[\widetilde{\mathbf{u}}, \widetilde{\mathbf{s}}, \widetilde{p}]$ depend on the fields $\left[\mathbf{u}_{h}, \mathbf{s}_{h}, p_{h}\right]$, which are interelement continuous.

Remark 6 The magnitude of the sub-grid scales decreases upon mesh refinement: as the finite element scale becomes finer, the residuals reduce. In other words, the sub-grid scales $[\widetilde{\mathbf{u}}, \widetilde{\mathbf{s}}, \widetilde{p}]$ are "small" compared to the finite element fields $\left[\mathbf{u}_{h}, \mathbf{s}_{h}, p_{h}\right]$.

Remark 7 It is interesting to observe that both the volumetric and the deviatoric components of the stress field are expressed as the "blending" of the continuous fields $\left(\mathbf{s}_{h}\right.$ and $\left.p_{h}\right)$ with the element-by-element discontinuous stress components, $\mathbb{C}^{\text {dev }}: \nabla^{s} \mathbf{u}_{h}$ and $\widetilde{C}^{v o l}\left(\nabla \cdot \mathbf{u}_{h}\right)$, respectively. The stability of the solution is ensured by adding those "small" discontinuous contributions to the continuous fields. 
Introducing the approximate fields $(78-80)$ into the original problem (59), the VMS stabilized formulation for of the compressible problem is obtained:

$$
\begin{aligned}
& {\left[\begin{array}{c}
\tau_{p} C^{v o l}\left(\nabla \cdot \mathbf{v}_{h}, \nabla \cdot \mathbf{u}_{h}\right)+ \\
\tau_{s}\left(\nabla^{s} \mathbf{v}_{h}, \mathbb{C}^{\text {dev }}: \nabla^{s} \mathbf{u}_{h}\right)
\end{array}\right]+\left(1-\tau_{s}\right)\left(\mathbb{P}: \nabla^{s} \mathbf{v}_{h}, \mathbf{s}_{h}\right) \quad+\quad\left(1-\tau_{p}\right)\left(\nabla \cdot \mathbf{v}_{h}, p_{h}\right) \quad F\left(\mathbf{v}_{h}\right)} \\
& \left(1-\tau_{s}\right)\left(\gamma_{h}, \mathbb{P}: \nabla^{s} \mathbf{u}_{h}\right) \quad-\left[\begin{array}{c}
\left(1-\tau_{s}\right)\left(\gamma_{h}, \mathbb{D}: \mathbf{s}_{h}\right)+ \\
\tau_{u}\left(\nabla \cdot \gamma_{h}, \nabla \cdot \mathbf{s}_{h}\right)
\end{array}\right] \quad-\quad \tau_{u}\left(\nabla \cdot \gamma_{h}, \nabla p_{h}\right) \quad=\quad 0 \\
& \left(1-\tau_{p}\right)\left(q_{h}, \nabla \cdot \mathbf{u}_{h}\right) \quad-\quad \tau_{u}\left(\nabla q_{h}, \nabla \cdot \mathbf{s}_{h}\right) \quad-\left[\begin{array}{c}
\left(1-\tau_{p}\right) D^{v o l}\left(q_{h}, p_{h}\right)+ \\
\tau_{u}\left(\nabla q_{h}, \nabla p_{h}\right)
\end{array}\right]=0
\end{aligned}
$$

The elemental stiffness matrix of problem (82) is expressed as:

$$
\mathbb{K}^{(e)}=\mathbb{K}_{h}^{(e)}-\tau_{u}^{(e)} \mathbb{K}_{\tau_{u}}^{(e)}-\tau_{s}^{(e)} \mathbb{K}_{\tau_{s}}^{(e)}-\tau_{p}^{(e)} \mathbb{K}_{\tau_{p}}^{(e)}
$$

which shows that there exist 3 different contributions adding stability to the original problem. The matrix form of these terms is:

$$
\begin{aligned}
\mathbb{K}_{\tau_{u}}^{(e)} & =\int_{\Omega^{(e)}}\left[\begin{array}{ccc}
{[\mathbf{0}]} & {[\mathbf{0}]} & {[\mathbf{0}]} \\
{[\mathbf{0}]} & {\left[\mathbf{B B}^{T}\right]} & {[\mathbf{B G}]} \\
{[\mathbf{0}]} & {\left[\mathbf{G}^{T} \mathbf{B}^{T}\right]} & {\left[\mathbf{G}^{T} \mathbf{G}\right]}
\end{array}\right] d \Omega \\
\mathbb{K}_{\tau_{s}}^{(e)} & =\int_{\Omega^{(e)}}\left[\begin{array}{ccc}
-\left[\mathbf{B}^{T} \mathbf{C}^{d e v} \mathbf{B}\right] & {\left[\mathbf{B}^{T} \mathbf{P} \mathbf{N}_{s}\right]} & {[\mathbf{0}]} \\
{\left[\mathbf{N}_{s}^{T} \mathbf{P B}\right]} & -\left[\mathbf{N}_{s}^{T} \mathbf{D} \mathbf{N}_{s}\right] & {[\mathbf{0}]} \\
{[\mathbf{0}]} & {[\mathbf{0}]} & {[\mathbf{0}]}
\end{array}\right] d \Omega \\
\mathbb{K}_{\tau_{p}}^{(e)} & =\int_{\Omega^{(e)}}\left[\begin{array}{ccc}
-C^{v o l}\left[\mathbf{G G}^{T}\right] & {[\mathbf{0}]} & {\left[\mathbf{G N} \mathbf{N}_{p}\right]} \\
{[\mathbf{0}]} & {[\mathbf{0}]} & {[\mathbf{0}]} \\
{\left[\mathbf{N}_{p}^{T} \mathbf{G}^{T}\right]} & {[\mathbf{0}]} & -D^{v o l}\left[\mathbf{N}_{p}^{T} \mathbf{N}_{p}\right]
\end{array}\right] d \Omega
\end{aligned}
$$

Observe that in the compressible case, taking $\tau_{p}=\tau_{s}$, the sub-matrix $\mathbb{K}_{u u}$, coming from the stabilization terms, can be re-written as:

$$
\mathbb{K}_{u u}=\tau_{s} C^{v o l} \mathbf{G G}^{T}+\tau_{s} \mathbf{B}^{T} \mathbf{C}^{\operatorname{dev}} \mathbf{B}=\tau_{s} \mathbf{B}^{T} \mathbf{C B}
$$

being the constitutive tensor (in Voigt's notation) defined by:

$$
\mathbf{C}=C^{v o l}(\mathbf{1} \otimes \mathbf{1})+\mathbf{C}^{d e v}
$$

Remark 8 Problem (82) is symmetric and definite even in the incompressible limit case. In this case, problem (82) reduces to:

$$
\begin{aligned}
& {\left[\begin{array}{c}
\tau_{p} \widetilde{C}^{v o l}\left(\nabla \cdot \mathbf{v}_{h}, \nabla \cdot \mathbf{u}_{h}\right)+ \\
\tau_{s}\left(\nabla^{s} \mathbf{v}_{h}, \mathbb{C}^{\text {dev }}: \nabla^{s} \mathbf{u}_{h}\right)
\end{array}\right]+\left(1-\tau_{s}\right)\left(\mathbb{P}: \nabla^{s} \mathbf{v}_{h}, \mathbf{s}_{h}\right) \quad+\quad\left(\nabla \cdot \mathbf{v}_{h}, p_{h}\right) \quad=F\left(\mathbf{v}_{h}\right)} \\
& \left(1-\tau_{s}\right)\left(\gamma_{h}, \mathbb{P}: \nabla^{s} \mathbf{u}_{h}\right) \quad-\left[\begin{array}{c}
\left(1-\tau_{s}\right)\left(\gamma_{h}, \mathbb{D}^{d e v}: \mathbf{s}_{h}\right)+ \\
\tau_{u}\left(\nabla \cdot \gamma_{h}, \nabla \cdot \mathbf{s}_{h}\right)
\end{array}\right]-\tau_{u}\left(\nabla \cdot \gamma_{h}, \nabla p_{h}\right)=\quad 0 \\
& \left(q_{h}, \nabla \cdot \mathbf{u}_{h}\right) \quad-\quad \tau_{u}\left(\nabla q_{h}, \nabla \cdot \mathbf{s}_{h}\right) \quad-\quad \tau_{u}\left(\nabla q_{h}, \nabla p_{h}\right) \quad=\quad 0
\end{aligned}
$$


which is symmetric and definite. Taking $\tau_{p}=\tau_{s}$, the sub-matrix $\mathbb{K}_{u u}$, can be re-written (for the linear elastic problem) as:

$$
\mathbb{K}_{u u}=\tau_{s} \widetilde{C}^{v o l} \mathbf{G G}^{T}+\tau_{s} \mathbf{B}^{T} \mathbf{C}^{\operatorname{dev}} \mathbf{B}=\tau_{s} 2 G \mathbf{B}^{T} \mathbf{B}
$$

\section{The mixed $u / \sigma$ formulation: the incompressible limit}

The three-field formulation assumes the pressure field as an independent variable of the problem. Doing this, the volumetric and the deviatoric components of the constitutive equation can be treated independently. The pressure field is interpolated separately from the deviatoric part of the stress tensor. This is particularly appealing in the incompressible limit, when the volumetric part of the constitutive equation transforms into a purely kinematic constraint of the problem, while conserving the accuracy induced by the nodal interpolation of the (deviatoric) stress field.

In this Section, the mixed $\mathbf{u} / \boldsymbol{\sigma}$ formulation is re-written to accomplish the incompressibility constraint. Staring from the strong format of the $\mathbf{u} / \boldsymbol{\sigma}$ formulation in $(5-6)$, and taking advantage from the split of the flexibility tensor into its volumetric and deviatoric components (see Eq. (36)), the result is:

$$
\begin{array}{r}
\nabla \cdot \boldsymbol{\sigma}+\mathbf{b}=\mathbf{0} \\
\nabla^{s} \mathbf{u}-\left(\mathbb{D}^{v o l}+\mathbb{D}^{d e v}\right): \boldsymbol{\sigma}=\mathbf{0}
\end{array}
$$

The corresponding weak form is:

$$
\begin{aligned}
& 0+\left(\nabla^{s} \mathbf{v}, \boldsymbol{\sigma}\right) \quad=F(\mathbf{v}) \\
& \left(\boldsymbol{\gamma}, \nabla^{s} \mathbf{u}\right)-\left(\frac{\operatorname{tr}(\boldsymbol{\gamma})}{3}, D^{v o l} \frac{\operatorname{tr}(\boldsymbol{\sigma})}{3}\right)-\left(\boldsymbol{\gamma}, \mathbb{D}^{d e v}: \boldsymbol{\sigma}\right)=0
\end{aligned}
$$

where $\mathbb{D}^{\text {dev }}:(\mathbb{P}: \boldsymbol{\sigma})=\mathbb{D}^{\text {dev }}: \boldsymbol{\sigma}$ because $\mathbb{P}$ is an idempotent operator.

Compared to the three-field formulation, here the pressure is computed as a function of the stress field as $\frac{\operatorname{tr}(\boldsymbol{\sigma})}{3}$, so that:

$$
\boldsymbol{\sigma}=\mathbb{V}: \boldsymbol{\sigma}+\mathbb{P}: \boldsymbol{\sigma}=\frac{\operatorname{tr}(\boldsymbol{\sigma})}{3} \mathbf{I}+\mathbf{s}(\boldsymbol{\sigma})
$$

The format in (93) is particularly convenient in the incompressible limit $\left(D^{v o l} \rightarrow 0\right)$. In this case, the problem reduces to:

$$
\begin{aligned}
& 0+\left(\nabla^{s} \mathbf{v}, \boldsymbol{\sigma}\right)=F(\mathbf{v}) \\
& \left(\boldsymbol{\gamma}, \nabla^{s} \mathbf{u}\right)-\left(\boldsymbol{\gamma}, \mathbb{D}^{\text {dev }}: \boldsymbol{\sigma}\right)=0
\end{aligned}
$$

Remark 9 The second term in the second equation of (95) is deviatoric so that this equation can be rewritten grouping the spheric and the deviatoric terms:

$$
\left(\boldsymbol{\gamma}, \frac{1}{3}(\nabla \cdot \mathbf{u}) \mathbf{I}\right)+\left(\boldsymbol{\gamma}, \mathbb{P}: \nabla^{s} \mathbf{u}-\mathbb{D}^{d e v}: \boldsymbol{\sigma}\right)=0
$$


As a consequence, the above equation is enforcing (in weak form) the deviatoric part of the constitutive equation as well as the incompressibility constraint, that is:

$$
\begin{aligned}
\mathbb{P}: \nabla^{s} \mathbf{u}-\mathbb{D}^{d e v}: \boldsymbol{\sigma} & =\mathbf{0} \\
\nabla \cdot \mathbf{u} & =0
\end{aligned}
$$

Remark 10 In problem (95), the volumetric part of the stress tensor, $\operatorname{tr}(\boldsymbol{\sigma})$, is determined up to a constant that need to be fixed through Neumann's boundary conditions.

Remark 11 The particularization to linear elasticity reads:

$$
\begin{array}{ccccc}
0 & + & \left(\nabla^{s} \mathbf{v}, \boldsymbol{\sigma}\right) & = & F(\mathbf{v}) \\
\left(\boldsymbol{\gamma}, \nabla^{s} \mathbf{u}\right) & - & \left(\boldsymbol{\gamma},\left(\frac{\mathbb{V}}{3 K}+\frac{\mathbb{P}}{2 G}\right): \boldsymbol{\sigma}\right) & = & 0
\end{array}
$$

and in the incompressible limit $(K \rightarrow \infty)$, it reduces to:

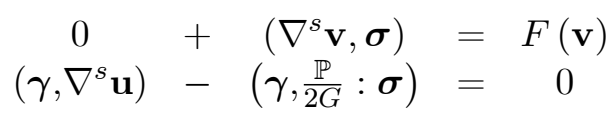

The discrete Galerkin finite element stiffness matrix is:

$$
\mathbb{K}_{h}^{(e)}=\int_{\Omega^{(e)}}\left[\begin{array}{cc}
{[\mathbf{0}]} & {\left[\mathbf{B}^{T} \mathbf{N}_{\sigma}\right]} \\
{\left[\mathbf{N}_{\sigma}^{T} \mathbf{B}\right]} & -\left[\mathbf{N}_{\sigma}^{T} \mathbf{D} \mathbf{N}_{\sigma}\right]
\end{array}\right] d \Omega
$$

where $\mathbf{N}_{\sigma}$ is a $6 \times 6 n_{\text {nod }}$ matrix made of $n_{\text {nod }}$ bocks of the form $N^{A} \mathbf{I}_{6 \times 6}$, being $N^{A}$ the shape functions of the selected element type of the FE discretization.

In the incompressible limit the element stiffness matrix reduces to:

$$
\mathbb{K}_{h}^{(e)}=\int_{\Omega^{(e)}}\left[\begin{array}{cc}
{[\mathbf{0}]} & {\left[\mathbf{B}^{T} \mathbf{N}_{\sigma}\right]} \\
{\left[\mathbf{N}_{\sigma}^{T} \mathbf{B}\right]} & -\left[\mathbf{N}_{\sigma}^{T} \mathbf{D}^{\operatorname{dev}} \mathbf{N}_{\sigma}\right]
\end{array}\right] d \Omega
$$

Remark 12 Both matrix formats (101) and (102) can be (formally) obtained summing the second and third rows and columns in (70) for the compressible and incompressible cases, respectively, when assuming the same (discrete) interpolation functions for both the pressure and the deviatoric stress fields.

Within the framework of the VMS method, the sub-grid scales for the displacement and stress fields are chosen as:

$$
\begin{aligned}
& \widetilde{\mathbf{u}}=\widetilde{\boldsymbol{\tau}}_{u} R_{u}^{h}=\widetilde{\boldsymbol{\tau}}_{u}\left[\nabla \cdot \boldsymbol{\sigma}_{h}+\mathbf{b}\right] \\
& \widetilde{\boldsymbol{\sigma}}=\widetilde{\boldsymbol{\tau}}_{\sigma} R_{s}^{h}= \begin{cases}\widetilde{\boldsymbol{\tau}}_{\sigma}:\left[\nabla^{s} \mathbf{u}_{h}-\mathbb{D}: \boldsymbol{\sigma}_{h}\right] & \text { Compressible case } \\
\widetilde{\boldsymbol{\tau}}_{\sigma}:\left[\nabla^{s} \mathbf{u}_{h}-\mathbb{D}^{d e v}: \boldsymbol{\sigma}_{h}\right] & \text { Incompressible limit }\left(D^{\text {vol }} \rightarrow 0\right)\end{cases}
\end{aligned}
$$

where the stabilization parameters are defined as:

$$
\begin{aligned}
& \widetilde{\boldsymbol{\tau}}_{u}=\tau_{u} \mathbf{I}_{3 \times 3} \quad \leftarrow \quad \tau_{u}=c_{u} \frac{L h}{2 \widehat{G}} \\
& \widetilde{\boldsymbol{\tau}}_{\sigma}=\tau_{\sigma} \widetilde{\mathbb{C}} \quad \leftarrow \quad \tau_{\sigma}=c_{\sigma} \frac{h}{L}
\end{aligned}
$$


Tensor $\widetilde{\mathbb{C}}=\widetilde{\mathbb{C}}^{v o l}+\mathbb{C}^{\text {dev }}$ (only used for stabilization purposes) is defined as:

$$
\widetilde{\mathbb{C}}=\widetilde{C}^{v o l}(\mathbf{I} \otimes \mathbf{I})+\mathbb{C}^{\text {dev }}=\left\{\begin{array}{cl}
\mathbb{C} & \text { Compressible case }\left(\widetilde{C}^{v o l}=C^{v o l}=K\right) \\
2 G \mathbb{I} & \text { Incompressible limit }\left(\widetilde{C}^{v o l}=\frac{2}{3} G\right)
\end{array}\right.
$$

This given, the solution of the problem is approximated as:

$$
\begin{aligned}
& \mathbf{u} \simeq \mathbf{u}_{h}+\widetilde{\mathbf{u}}=\mathbf{u}_{h}+\tau_{u}\left[\nabla \cdot \boldsymbol{\sigma}_{h}+\mathbf{b}\right] \\
& \boldsymbol{\sigma} \simeq \boldsymbol{\sigma}_{h}+\tilde{\boldsymbol{\sigma}}=\left\{\begin{array}{lll}
\left(1-\tau_{\sigma}\right) & \boldsymbol{\sigma}_{h}+\tau_{\sigma}\left[\mathbb{C}: \nabla^{s} \mathbf{u}_{h}\right] & \text { Compressible case } \\
\left(1-\tau_{\sigma}\right) & \boldsymbol{\sigma}_{h}+\tau_{\sigma}\left[2 G \nabla^{s} \mathbf{u}_{h}\right] & \text { Incompressible limit }
\end{array}\right.
\end{aligned}
$$

and the corresponding stabilized problem for the compressible case is:

$$
\begin{aligned}
& \tau_{\sigma}\left(\nabla^{s} \mathbf{v}_{h}, \mathbb{C}: \nabla^{s} \mathbf{u}_{h}\right)+\left(1-\tau_{\sigma}\right)\left(\nabla^{s} \mathbf{v}_{h}, \boldsymbol{\sigma}_{h}\right) \quad=F\left(\mathbf{v}_{h}\right) \\
& \left(1-\tau_{\sigma}\right)\left(\gamma_{h}, \nabla^{s} \mathbf{u}_{h}\right)-\left[\begin{array}{c}
\left(1-\tau_{\sigma}\right)\left(\gamma_{h}, \mathbb{D}: \boldsymbol{\sigma}_{h}\right)+ \\
\tau_{u}\left(\nabla \cdot \gamma_{h}, \nabla \cdot \boldsymbol{\sigma}_{h}\right)
\end{array}\right]=0
\end{aligned}
$$

The elemental stiffness matrix can be expressed as:

$$
\mathbb{K}^{(e)}=\mathbb{K}_{h}^{(e)}-\tau_{u}^{(e)} \mathbb{K}_{\tau_{u}}^{(e)}-\tau_{\sigma}^{(e)} \mathbb{K}_{\tau \sigma}^{(e)}
$$

which shows that there exist two different contributions adding stability to the original Galerkin's problem. These contributions are:

$$
\begin{aligned}
\mathbb{K}_{\tau_{u}}^{(e)} & =\int_{\Omega^{(e)}}\left[\begin{array}{cc}
{[\mathbf{0}]} & {[\mathbf{0}]} \\
{[\mathbf{0}]} & {\left[\mathbf{B B}^{T}\right]}
\end{array}\right] d \Omega \\
\mathbb{K}_{\tau_{\sigma}}^{(e)} & =\int_{\Omega^{(e)}}\left[\begin{array}{cc}
-\left[\mathbf{B}^{T} \mathbf{C B}\right] & {\left[\mathbf{B}^{T} \mathbf{N}_{\sigma}\right]} \\
{\left[\mathbf{N}_{\sigma}^{T} \mathbf{B}\right]} & -\left[\mathbf{N}_{\sigma}^{T} \mathbf{D} \mathbf{N}_{\sigma}\right]
\end{array}\right] d \Omega
\end{aligned}
$$

In the incompressible limit, the stabilized problem reads:

$$
\begin{gathered}
\tau_{\sigma} 2 G\left(\nabla^{s} \mathbf{v}_{h}, \nabla^{s} \mathbf{u}_{h}\right)+\frac{\left(1-\tau_{\sigma}\right)\left(\nabla^{s} \mathbf{v}_{h}, \boldsymbol{\sigma}_{h}\right)}{+}=F\left(\mathbf{v}_{h}\right) \\
\left(1-\tau_{\sigma}\right)\left(\gamma_{h}, \nabla^{s} \mathbf{u}_{h}\right)-\left[\begin{array}{c}
\left(1-\tau_{\sigma}\right)\left(\gamma_{h}, \mathbb{D}^{d e v}: \boldsymbol{\sigma}_{h}\right)+ \\
\tau_{u}\left(\nabla \cdot \gamma_{h}, \nabla \cdot \boldsymbol{\sigma}_{h}\right)
\end{array}\right]=0
\end{gathered}
$$

and the corresponding stabilization matrices are:

$$
\begin{aligned}
\mathbb{K}_{\tau_{u}}^{(e)} & =\int_{\Omega^{(e)}}\left[\begin{array}{cc}
{[\mathbf{0}]} & {[\mathbf{0}]} \\
{[\mathbf{0}]} & {\left[\mathbf{B B}^{T}\right]}
\end{array}\right] d \Omega \\
\mathbb{K}_{\tau_{\sigma}}^{(e)} & =\int_{\Omega^{(e)}}\left[\begin{array}{cc}
-2 G\left[\mathbf{B}^{T} \mathbf{B}\right] & {\left[\mathbf{B}^{T} \mathbf{N}\right]} \\
{\left[\mathbf{N}^{T} \mathbf{B}\right]} & -\left[\mathbf{N}^{T} \mathbf{D}^{d e v} \mathbf{N}\right]
\end{array}\right] d \Omega
\end{aligned}
$$


Remark 13 The stabilization matrices for both the compressible and incompressible cases can be (formally) obtained summing the second and third rows and columns of the corresponding matrices defined for the three-field formulation, and assuming the same (discrete) interpolation functions for both the pressure and the deviatoric stress fields as well as the same stabilization parameters: $\tau_{\sigma}=\tau_{s}=\tau_{p}$. Thus, the $\mathbf{u} / \boldsymbol{\sigma}$ formulation can be considered a particular case of the $\mathbf{u} / \mathbf{s} / p$ formulation. The latter allows one to approximate independently the deviatoric and volumetric parts of the stress, whereas in the former they are limited by the stress approximation chosen.

\section{Numerical results}

In this section, the mixed three-field formulation presented in this work is tested in a number of numerical benchmarks. The objective is to show the performance of the proposed finite element technology in terms of both displacement and stress field accuracy and its rate of convergence upon mesh refinement.

For the sake of brevity, the incompressible linear elasticity case is studied, although the results can be extended to more complex non-linear constitutive behaviors (allowing for the volumetric/deviator split).

Full incompressibility (Poisson's ratio: $\nu=0.5$ ) is assumed for the different numerical tests. The performance of the proposed method is compared with the behavior of the mixed displacement/pressure formulation (see [20]). The same displacement sub-grid scale stabilization $\left(\tau_{u}=\right.$ $c_{u} \frac{h^{2}}{2 G}$ with $\left.c_{u}=1.0\right)$ is adopted for both the $\mathbf{u} / p$ and $\mathbf{u} / \mathbf{s} / p$ formulations, while the stress sub-grid has been introduced in the $\mathbf{u} / \mathbf{s} / p$ formulation assuming: $\tau_{s}=c_{s} \frac{h}{L}$ with $c_{s}=1$ and $\tau_{p}=0$.

Calculations have been performed with an enhanced version of the finite element code COMET (see [12]) developed by the authors at the International Center for Numerical Methods in Engineering (CIMNE).

\subsection{Plane strain Cook's membrane problem}

The Cook's membrane problem is a bending dominated benchmark used by many authors to test their element formulations (see [34], [19], among others). The problem consists of a tapered panel, clamped on the left hand side and subjected to a shearing load $(f=1)$ at the right end. Initial geometry of this plane strain problem is shown in Figure 1. Material data have been assigned in terms of Young's modulus: $E=200$ and Poisson's ratio: $\nu=0.5$. For the evaluation of the stabilization parameters in the mixed $\mathbf{u} / \mathbf{s} / p$ formulation the characteristic length is taken equal to 50 .

In order to test the convergence behavior of the different formulations, the problem has been discretized into a series of regular mesh refinements with $2 \times 2,4 \times 4,8 \times 8,16 \times 16,32 \times 32,64 \times 64$, $128 \times 128$ and $256 \times 256$ elements per side. Both structured quadrilateral and triangular meshes have been tested. In Figure 2 the $16 \times 16$ quadrilateral and triangular meshes are shown.

Figure 3 compares the performance of the $\mathbf{u} / \mathbf{s} / p$ vs. $\mathbf{u} / p$ formulations. It shows how they both converge to the reference values (best value obtained with the $256 \times 256$ quadrilateral mesh using the $\mathbf{u} / \mathbf{s} / p$ formulation) on mesh refinement. The performance is monitored reporting the displacement of the top right corner (point $A$ in Figure 1) and both the J2 deviatoric stress and pressure value at the mid point of the bottom side of the Cook's membrane (point $B$ in Figure 1). Note that the 


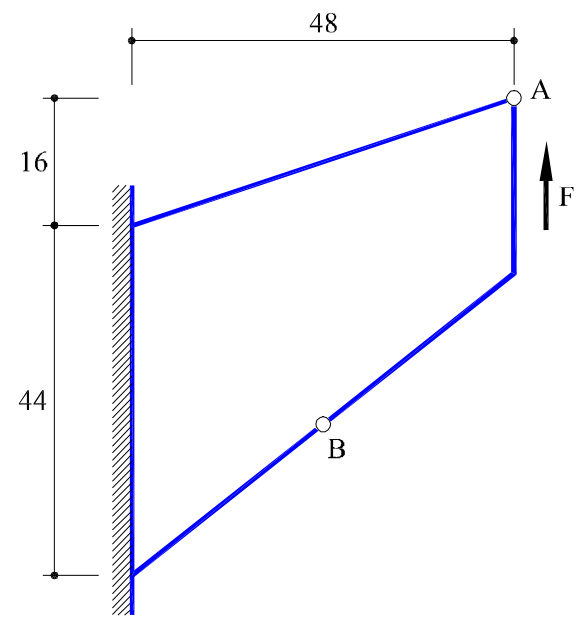

Figure 1: Cook's membrane problem: geometry.

$\mathbf{u} / p$ formulation computes the stresses (locally) at the Gauss points (Figure 4 (a)) while the $\mathbf{u} / \mathbf{s} / p$ formulation adopts a continuous stress field (Figure 4 (b)). To compare stress accuracy, a local smoothing technique has been applied to the original discontinuous stress fields of the mixed $\mathbf{u} / p$ formulation. So, Figure 3 presents the continuous values obtained after the smoothing operation.

Results in Figure 3 clearly show that both the $\mathbf{u} / \mathbf{s} / p$ and the $\mathbf{u} / p$ formulations deal appropriately with the incompressibility constraint but the three-field formulation exhibits a higher convergence rate in the stress fields. This translates into an enhanced stress accuracy of the proposed formulation even for very coarse meshes. The same considerations apply for both triangular and quadrilateral mesh discretizations.

For the sake of completeness, Figure 3 also shows results obtained for the bilinear displacement/constant pressure Q1P0 element ([33]) and the bilinear displacement with enhanced strains Q1E4 element ([34]). These two elements can only approach the incompressible limit, so a value of Poisson's ratio $\nu=0.4999$ has been used. The same local smoothing technique has been applied to the discontinuous stress fields that these elements produce. Both quadrilaterals perform satisfactorily on these regular structured meshes, showing an accuracy comparable to that of the mixed $\mathbf{u} / \mathbf{s} / p$ formulation. However. the finite element formulations behind these two elements cannot be extended to triangular and tetrahedral meshes.

\subsection{Cantilever beam}

Let us now consider a beam of unit thickness (plane-strain analysis) subjected to a bending moment imposed at right end side by means of a linear normal traction distribution (the maximum traction values is $f=10$ ), as shown in Fig. 5 .

Both the horizontal and vertical displacements are prescribed at the bottom corner of the left hand side. The horizontal displacement is also fixed at the upper corner. The exact solution of this 


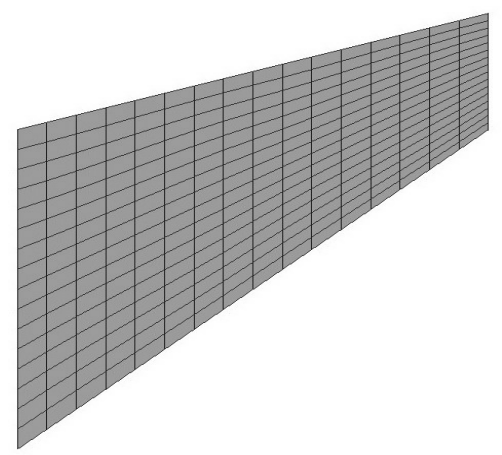

(a) Structured quadrilateral mesh

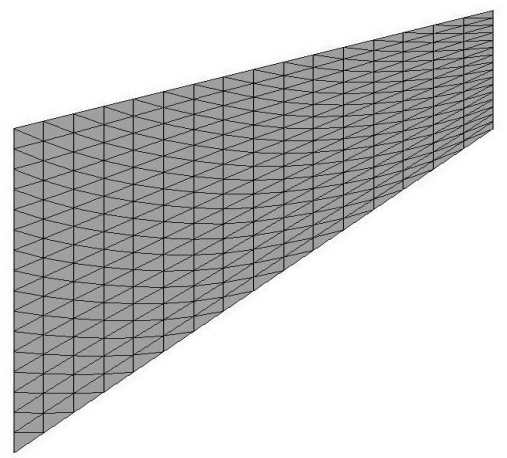

(b) Structured triangular mesh

Figure 2: Cook's membrane problem: (16 x 16) FE meshes.

pure bending problem is given by:

$$
\begin{aligned}
& u(x, y)=\frac{2 f\left(1-\nu^{2}\right)}{E L} x\left(\frac{L}{2}-y\right) \\
& v(x, y)=\frac{f\left(1-\nu^{2}\right)}{E L}\left[x^{2}+\frac{\nu}{1-\nu} y(y-L)\right]
\end{aligned}
$$

where $u(x, y)$ and $v(x, y)$ are the horizontal and vertical displacements $(0 \leq x \leq L$ and $0 \leq y \leq H)$.

The beam length is $L=10$ and its height is $H=2$. Young's modulus is set to $E=200$ and Poisson's ratio to $\nu=0.5$. For the evaluation of the stabilization parameters in the mixed $\mathbf{u} / \mathbf{s} / p$ formulation the characteristic length is taken equal to $H$.

To assess the accuracy of the proposed formulation, the vertical displacement at the top corner $(A)$ of the right side of the beam is monitored (the analytical value is $v(10,2)=0.375$ ), as well as the maximum horizontal normal stress and the mean stress (pressure) at the mid-point $(B)$ of the bottom side (the analytical values are $\sigma_{x x}=2$ and $p=1$, respectively).

The computational domain has been discretized into a series of orthogonal refined meshes with $1 \times 5,2 \times 10,4 \times 20,10 \times 50$ and $100 \times 500$ quadrilateral elements (see Fig. 6)

The performance of the proposed three-field formulation is shown in Figure 7 and compared to that of the displacement/pressure formulation. The enhanced accuracy of the proposed formulation is clearly demonstrated. Using very coarse meshes such as the $2 \times 10$ mesh (only 2 elements in the thickness), the error is less than $5 \%$ for the top corner displacement and less that $1 \%$ for both horizontal stress and pressure values.

Figure 8 depicts the vertical displacement along the top side of the beam using different mesh resolutions for both the $\mathbf{u} / \mathbf{s} / p$ and $\mathbf{u} / p$ formulations. The analytical solution is given by the parabolic function:

$$
v(x, 2)=\frac{f\left(1-\nu^{2}\right)}{E L} x^{2}=0.375 \cdot 10^{-2} x^{2}
$$




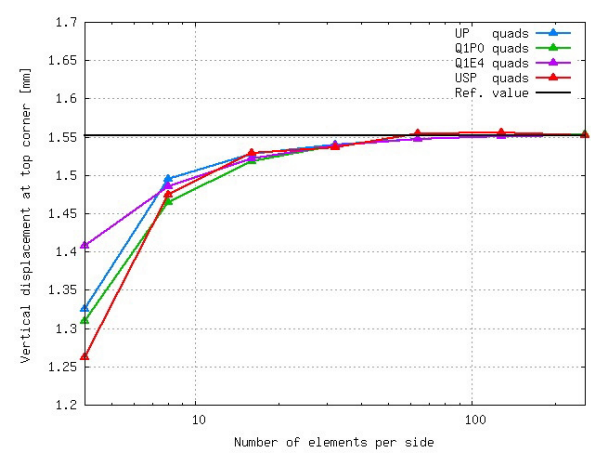

(a) QUAD: displacement

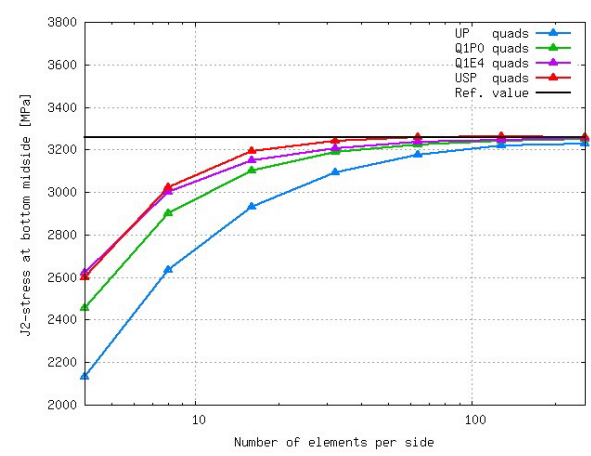

(c) QUAD: J2-stress

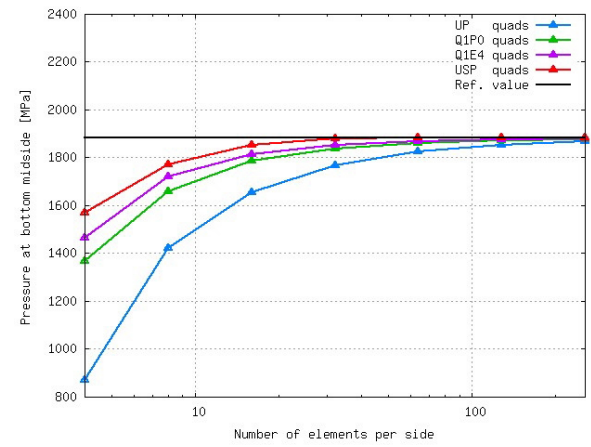

(e) QUAD: pressure

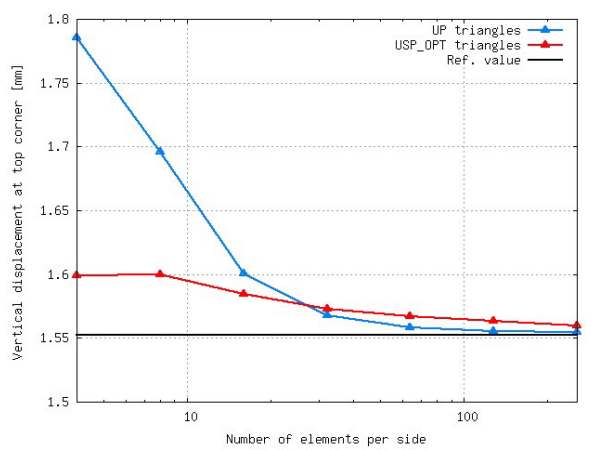

(b) TRI: displacement

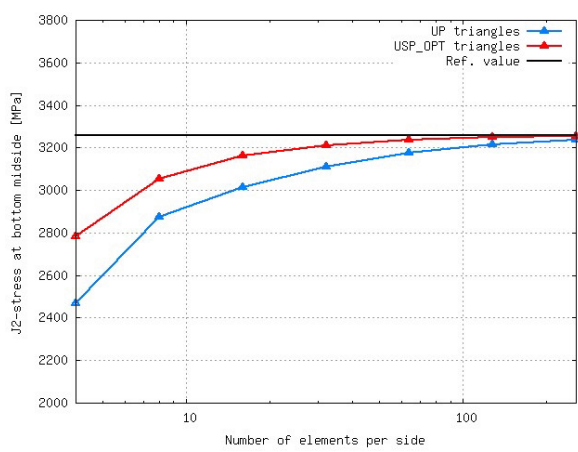

(d) TRI: J2-stress

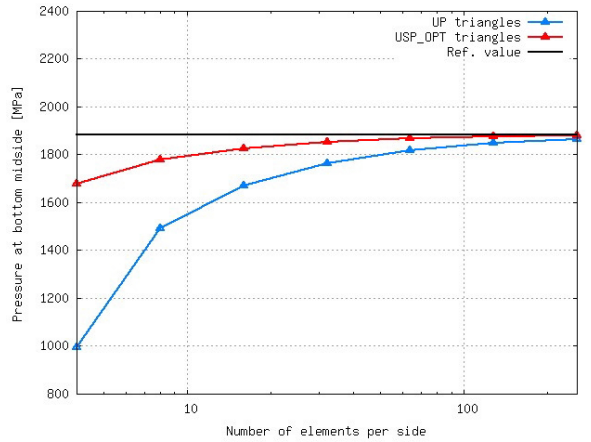

(f) TRI: pressure

Figure 3: Cook's membrane problem: performance of the proposed mixed three-field formulation compared with the mixed displacement/pressure formulation. 


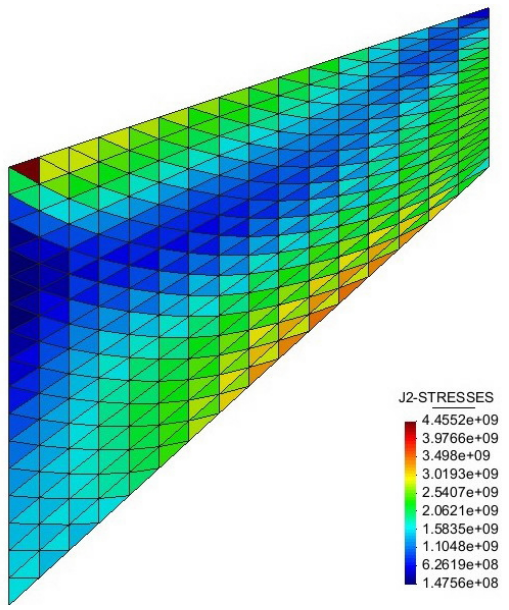

(a) $\mathbf{u} / \mathrm{p}$ formulation

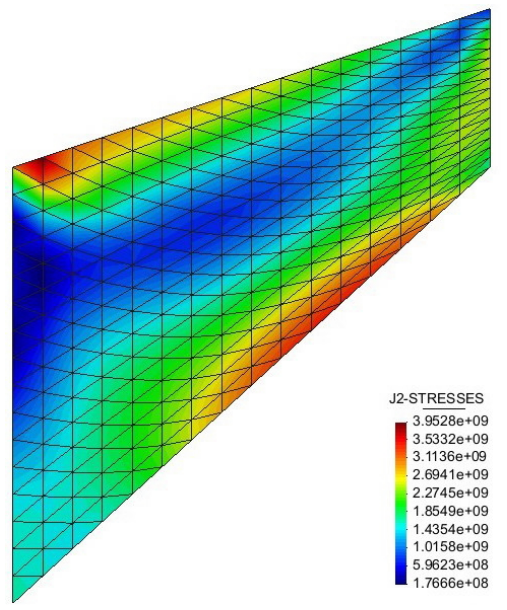

(b) $\mathbf{u} / \mathbf{s} / \mathrm{p}$ formulation

Figure 4: Cook's membrane problem (16x16 triangular mesh): J2 deviatoric stress value.

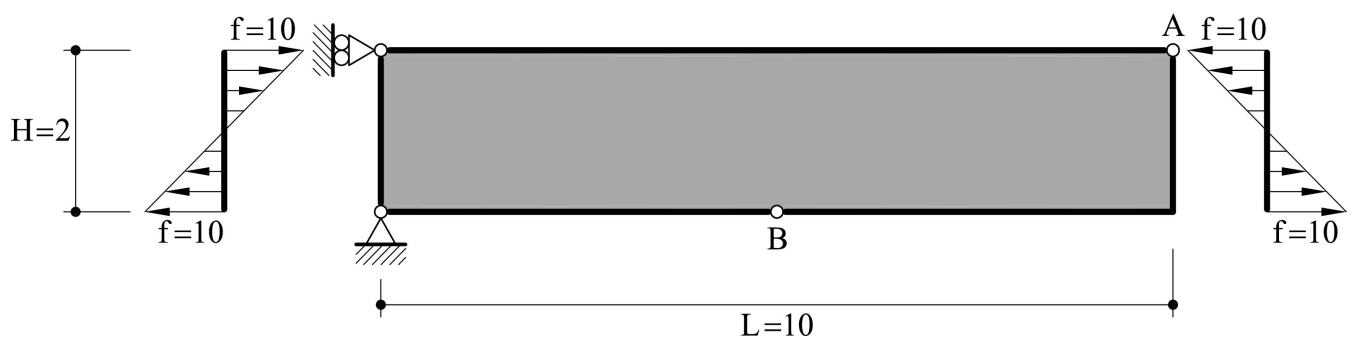

Figure 5: Cantilever beam: geometry.

Once again, the behavior of the proposed formulation shows a great degree of accuracy even for very coarse meshes.

Sensibility of the proposed formulation against element distortion is tested by comparing the above results on orthogonal meshes with those obtained on slanted meshes (see Fig. 6). Table 1 compares errors on maximum vertical displacements, horizontal normal stress and pressure obtained with the $\mathbf{u} / p$ and $\mathbf{u} / \mathbf{s} / p$ formulations on the $10 \times 50$ orthogonal and slanted meshes. The accuracy of the proposed $\mathbf{u} / \mathbf{s} / p$ formulation is almost insensitive to distortion. This is not the case for $\mathbf{u} / p$ formulation, where accuracy on stress and pressure deteriorates with slanting.

\subsection{Sharp V-notched specimen under tension}

As a last example, the vertical stretching of a square V-notched specimen, shown in Figure 9, is considered. Dimensions of the sample are $2 \times 2$ (width $\times$ height) and the V-shaped notch has a 


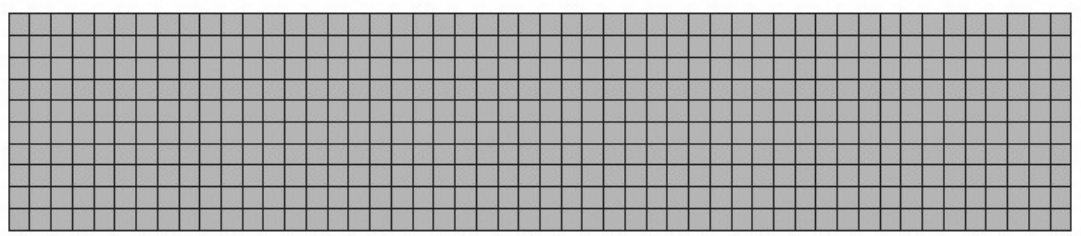

(a) Orthogonal mesh

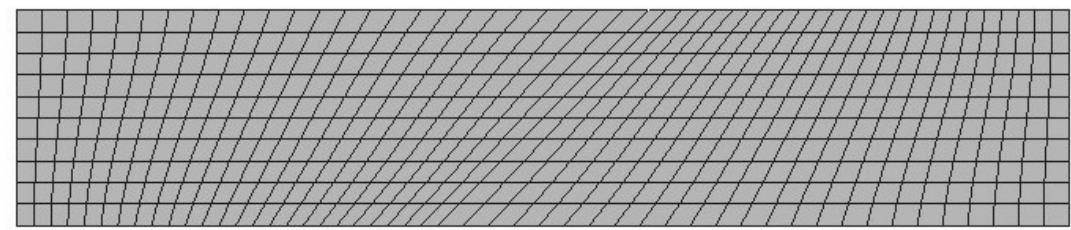

(b) Slanted mesh

Figure 6: Cantilever beam: 10x50 meshes.

\begin{tabular}{|l|c|c|c|}
\hline \hline & Max vertical displ. & Max horiz. stress & Max pressure \\
\hline $\mathbf{u} / p$ orth. & $+10.6 \%$ & $-0.30 \%$ & $-8.95 \%$ \\
\hline $\mathbf{u} / p$ slanted & $+2.03 \%$ & $-13.0 \%$ & $-12.43 \%$ \\
\hline $\mathbf{u} / \mathbf{s} / p$ orth. & $-0.26 \%$ & $+0.55 \%$ & $+3.14 \%$ \\
\hline $\mathbf{u} / \mathbf{s} / p$ slanted & $-0.53 \%$ & $+0.35 \%$ & $+1.74 \%$ \\
\hline
\end{tabular}

Table 1: Cantilever beam. Accuracy on orthogonal and slanted 10x50 meshes. 


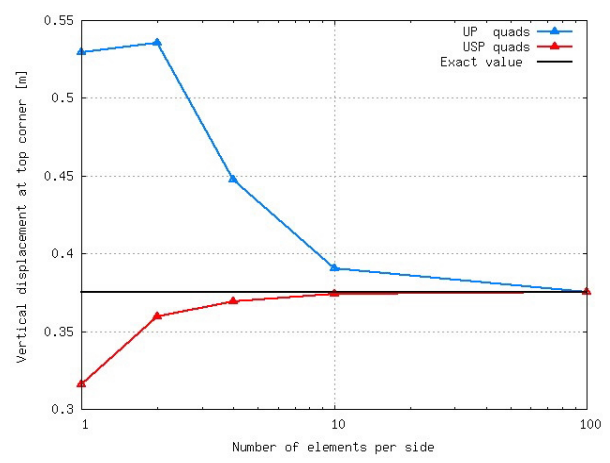

(a) Displacement

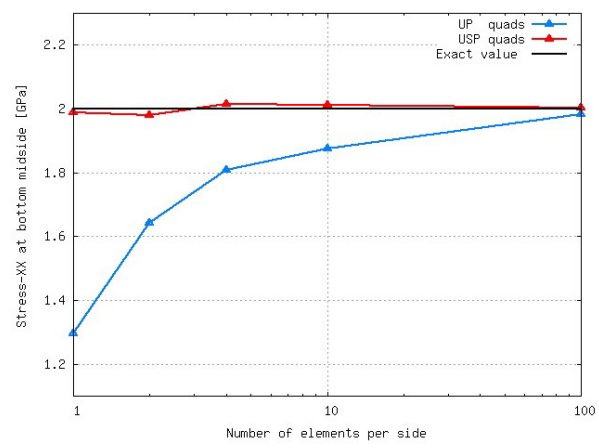

(b) Max. hor. normal stress

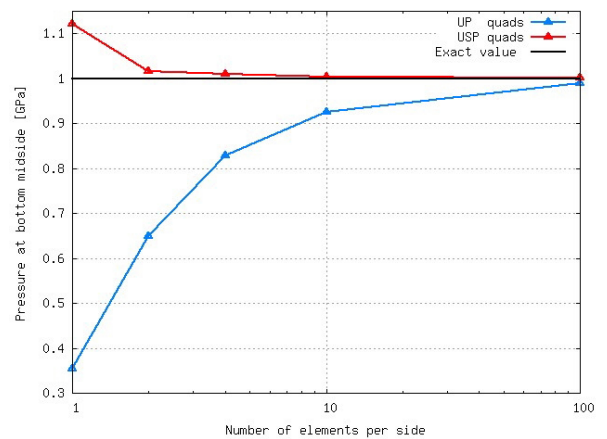

(c) Pressure

Figure 7: Cantilever beam: performance of the proposed mixed three-field formulation compared with the mixed displacement/pressure formulation. 


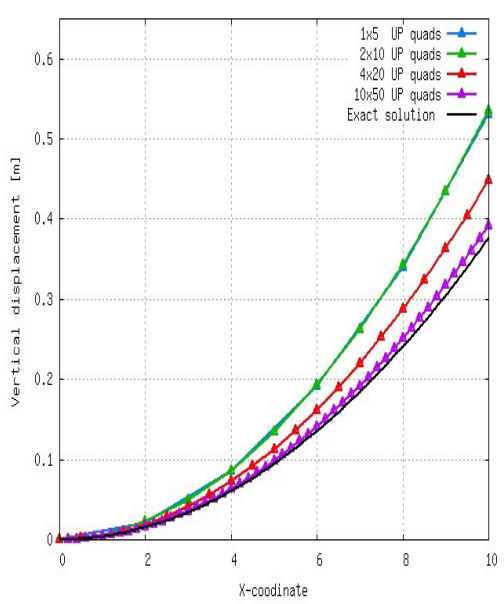

(a) $\mathbf{u} / \mathrm{p}$ formulation

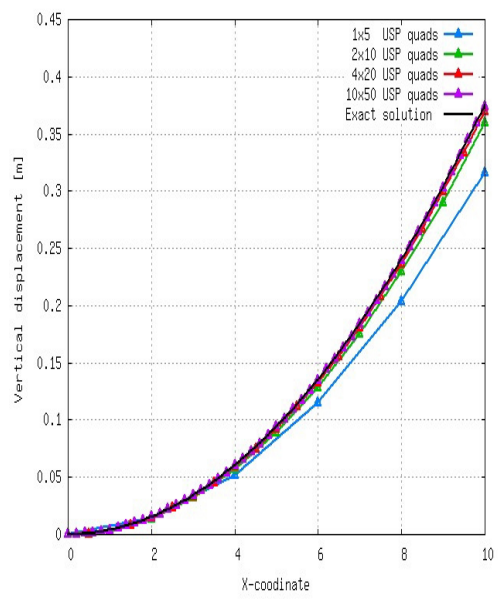

(b) $\mathbf{u} / \mathbf{s} / \mathrm{p}$ formulation

Figure 8: Cantilever beam problem: vertical displacement along the top side of the beam using different mesh resolutions.

length of 1 and a maximum width at the boundary of 0.02 . For the evaluation of the stabilization parameters in the mixed formulation, $L=2$ is taken as representative length of the problem. Uniform vertical displacements of opposed sign are imposed at the top and bottom boundaries.

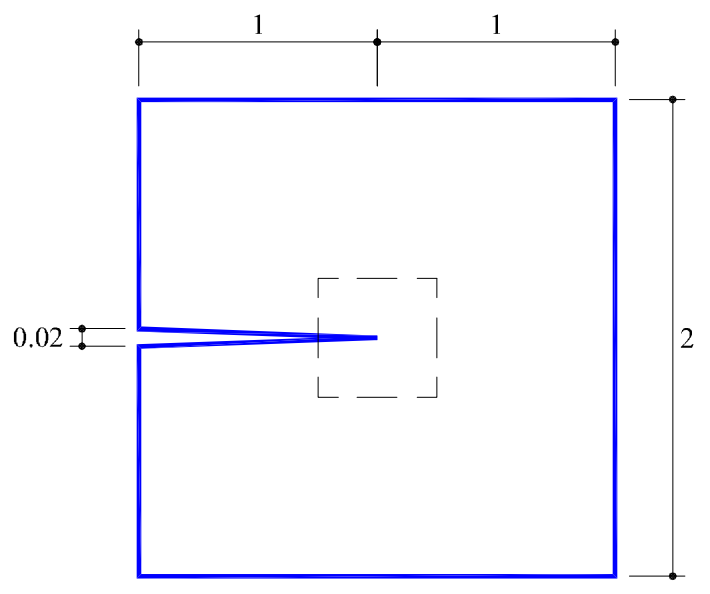

Figure 9: Geometry for the sharp V-notched specimen under tension

In the continuous elastic problem associated to this situation, the strain and stress fields are singular at the tip of the sharp notch. The discrete model corresponding to the $\mathbf{u} / p$ finite element formulation performs satisfactorily in terms of a global error norm, but approximates very poorly the actual behavior near the singular points.

To show this, a coarse structured mesh consisting of $8 \times 8 \times 2 \mathbf{u} / p$ triangles with a $\pm 45^{\circ}$ bias is 


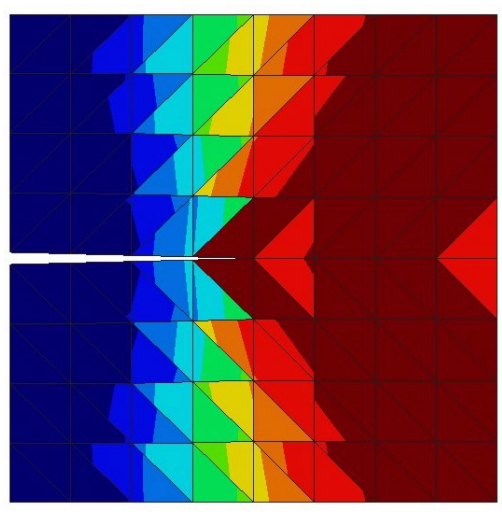

(a) $\mathrm{u} / \mathrm{p}$ formulation: max. pr. stress contour-fill.

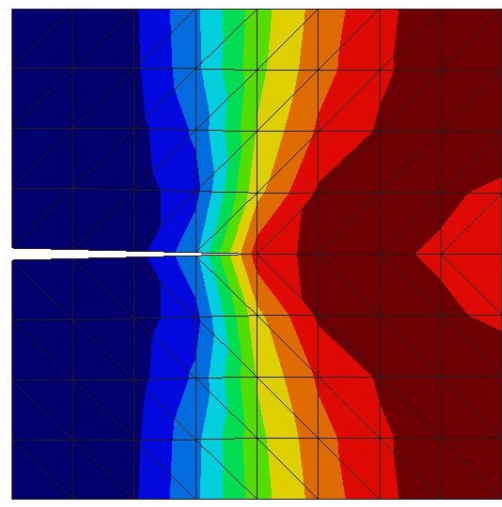

(c) $\mathrm{u} / \mathrm{s} / \mathrm{p}$ formulation: $\max$. pr. stress contour-fill.

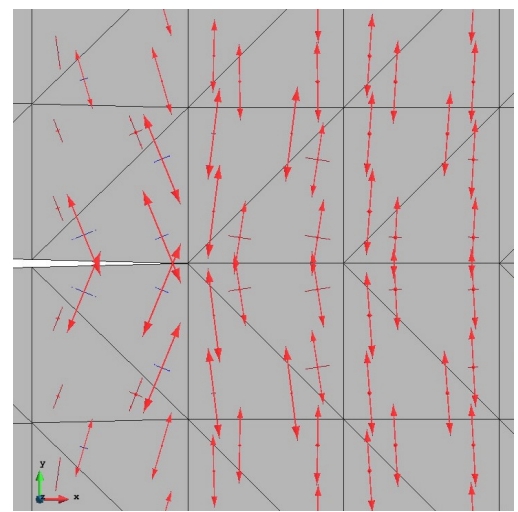

(b) $\mathrm{u} / \mathrm{p}$ formulation: principal stresses.

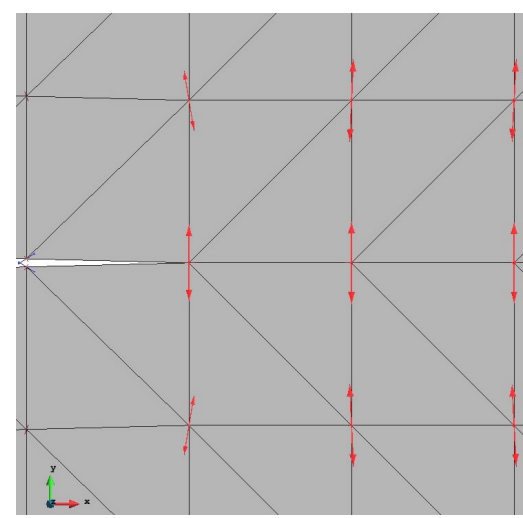

(d) $\mathrm{u} / \mathrm{s} / \mathrm{p}$ formulation: principal stresses.

Figure 10: Sharp V-notch specimen under tension.Principal stresses.

constructed. Figure 10(a) depicts contour-fill of the maximum principal stresses computed on this mesh. Note the strong mesh bias dependence that is observed in front of and behind the notch tip. In fact, the largest values of the stresses occur behind the tip (left of the tip in the Figures), rather than in front of it (right of the tip in the Figures). Computed stress directions near the tip of the crack also show strong mesh bias dependence (see Figure 10(b)). These severe local errors caused by the mesh alignment are not alleviated by mesh refinement.

Figures 10(c)-(d) show corresponding results obtained using the stabilized mixed $\mathbf{u} / \mathbf{s} / p$ formulation on the same mesh. The improved accuracy with respect to the $\mathbf{u} / p$ formulation is clear. In particular, the maximum principal stress value is detected exactly at the tip of the notch; computed stresses directions are also noticeably improved. The importance of these two features in nonlinear solid and fluid mechanics is evident. As it is shown in reference [18], they are crucial in strain 
localization problems where the constitutive equation depends on the principal stress values and their directions.

\section{Concluding remarks}

This paper shows that it is possible to tackle problems involving the incompressibility constraint (including the limit case of Poisson's ratio $\nu=0.5$ ) while, at the same time, achieving a remarkable degree of accuracy of the stress field.

The proposed three-field formulation is convergent upon mesh refinement, virtually free of any volumetric or shear locking. The technology is suitable for engineering applications in $2 \mathrm{D}$ and $3 \mathrm{D}$ and for both triangular (tetrahedral for 3D analysis) or quadrilateral (or hexahedral in 3D) meshes.

Numerical examples show the remarkable degree of accuracy (even for coarse meshes) for both displacement and stress fields.

The proposed element technology can easily be extended to more complex non-linear material behaviors allowing for the spheric/deviatoric split of the constitutive equations, such as incompressible J2-plasticity.

\section{Acknowledgments}

Financial support from the EC $7^{\text {th }}$ Framework Programme under the MuMoLaDe project - Multiscale Modelling of Landslides and Debris Flows - within the framework of Marie Curie ITN (Initial Training Networks) is gratefully acknowledged.

R. Codina gratefully acknowledges the support received from the ICREA Academia Program from the Catalan Government.

\section{References}

[1] Agelet de Saracibar C., Chiumenti M., Valverde Q., and Cervera M., On the Orthogonal Subgrid Scale Pressure stabilization of Small and Finite deformation J2 Plasticity, Computer Methods in Applied Mechanics and Engineering, 195 (2006) 1224-1251.

[2] Arnold, D.N., Brezzi, F. and Fortin, M. A stable finite element for the Stokes equations. Calcolo (1984) 21, 337-344.

[3] Arnold, D.N. and Winther, R. Mixed finite elements for elasticity, Numerische Mathematik (2002) 92, 401-419.

[4] Arnold, D.N., Awanou, G. , and Winther, R. Finite elements for symmetric tensors in three dimensions, Math. Comput. (2008) 77, 1229-1251.

[5] Badia, S. and Codina, R. Unified stabilized finite element formulations for the Stokes and the Darcy problems, SIAM Journal on Numerical Analysis (2009) 17, 309-330.

[6] Badia, S. and Codina, R. Stabilized continuous and discontinuous Galerkin techniques for Darcy flow, Comp. Meth. in Appl. Mech. and Eng. (2010) 199, 1654-1667. 
[7] Baiocchi, C., Brezzi, F. and Franca, L. Virtual bubbles and Galerkin/least-squares type methods (Ga.L.S.), Comp. Meth. in Appl. Mech. and Eng. (1993) 105, 125-141.

[8] Bonet, J. and Burton, A.J. A simple average nodal pressure tetrahedral element for incompressible and nearly incompressible dynamic explicit applications. Comm. Num. Meths. in Eng. (1998)1 4, 437-449.

[9] Bonet, J., Marriot, H. and Hassan, O. An averaged nodal deformation gradient linear tetrahedral element for large strain explicit dynamic applications. Comm. Num. Meths. in Eng. (2001) 17, 551-561.

[10] Bonet, J., Marriot, H. and Hassan, O. Stability and comparison of different linear tetrahedral formulations for nearly incompressible explicit dynamic applications. Int. Jour. for Num. Meths. in Eng. (2001) 50, 119-133.

[11] Brezzi, F. and Fortin, M. Mixed and Hybrid Finite Element Methods, Spinger, New York, 1991.

[12] Cervera, M., Agelet de Saracibar, C. and Chiumenti, M. COMET: COupled MEchanical and Thermal analysis. Data Input Manual, Version 5.0, Technical report IT-308, htpp://www.cimne.upc.es, 2002.

[13] Cervera, M., Chiumenti, M., Valverde, Q. and Agelet de Saracibar, C. Mixed Linear/linear Simplicial Elements for Incompressible Elasticity and Plasticity, Comp. Meth. in Appl. Mech. and Eng. (2003) 192, 5249-5263.

[14] Cervera, M., Chiumenti, M. and Agelet de Saracibar, C. Softening, localization and stabilization: capture of discontinuous solutions in J2 plasticity, Int. J. for Num. and Anal. Meth. in Geomechanics (2004) 28, 373-393.

[15] Cervera, M., Chiumenti, M. and Agelet de Saracibar, C. Shear band localization via local $\mathrm{J}_{2}$ continuum damage mechanics, Comp. Meth. in Appl. Mech. and Eng. (2004) 193, 849-880.

[16] Cervera, M. and Chiumenti, M. Size effect and localization in $\mathrm{J}_{2}$ plasticity, Int. J. of Solids and Structures (2009) 46, 3301-3312.

[17] Cervera, M., Chiumenti, M. and Codina, R. Mixed stabilized finite element methods in nonlinear solid mechanics. Part I: formulation, Comp. Meth. in Appl. Mech. and Eng., 199 (2010) 2559-2570.

[18] Cervera, M., Chiumenti, M. and Codina, R. Mixed stabilized finite element methods in nonlinear solid mechanics. Part II: strain localization, Comp. Meth. in Appl. Mech. and Eng., 199 (2010) 2571-2589.

[19] Chama, A. and Reddy, D.B. New stable mixed finite element approximations for problems in linear elasticity, Comput. Methods Appl. Mech. Eng. 256 (2013) 211-223. 
[20] Chiumenti, M., Valverde, Q., Agelet de Saracibar, C. and Cervera, M. A stabilized formulation for incompressible elasticity using linear displacement and pressure interpolations, Comp. Meth. in Appl. Mech. and Eng. (2002) 191, 5253-5264.

[21] Chiumenti, M., Valverde, Q., Agelet de Saracibar, C. and Cervera, M. A stabilized formulation for incompressible plasticity using linear triangles and tetrahedra, Int. J. of Plasticity (2004) $20,1487-1504$.

[22] Codina, R. Stabilization of incompressibility and convection through orthogonal sub-scales in finite element methods, Comp. Meth. in Appl. Mech. and Eng. (2000) 190, 1579-1599.

[23] Codina, R. Finite element approximation of the three field formulation of the Stokes problem using arbitrary interpolations, SIAM Journal on Numerical Analysis (2009) 47, 699-718.

[24] Dohrmann, C.R., Heinstein, M.W., Jung, J., Key, S.W. and Witkowsky, W.R. Node-based uniform strain elements for three-node triangular and four-node tetrahedral meshes. Int. Jour. for Num. Meths. in Eng. (2000) 47, 1549-1568.

[25] GiD: The personal pre and post preprocessor. htpp://www.gid.cimne.upc.es, 2002.

[26] Hughes, T.J.R. Multiscale phenomena: Green's function, Dirichlet-to Neumann formulation, subgrid scale models, bubbles and the origins of stabilized formulations, Comp. Meth. in Appl. Mech. and Eng. (1995) 127, 387-401.

[27] Hughes, T.J.R., Feijoó, G.R., Mazzei. L., Quincy, J.B. The variational multiscale method-a paradigm for computational mechanics, echanics, Comp. Meth. in Appl. Mech. and Eng. (1998) $166,3-28$.

[28] Kasper, E. P. and Taylor, R. L. A mixed-enhanced strain method. I: Geometrically linear problems. II: Geometrically nonlinear problems, Comput. Struct. 75 (2000) 237-250, 251-260.

[29] Malkus, D.S. and Hughes, T.J.R. Mixed finite element methods - reduced and selective integration techniques: a unification of concepts, Comp. Meth. in Appl. Mech. and Eng. (1978) $15,63-81$.

[30] Mijuca, D. On hexahedral finite element HC8/27 in elasticity, Computational Mechanics (2004) $33,466-480$.

[31] Pastor, M., Li, T., Liu, X. and Zienkiewicz, O.C. Stabilized low-order finite elements for failure and localization problems in undrained soils and foundations, Comp. Meth. in Appl. Mech. and Eng. (1999), 174, 219-234.

[32] Reddy, B.D. and Simo, J.C. Stability and convergence of a class of enhanced assumed strain methods, SIAM J. Num. Anal. (1995) 32, 1705-1728.

[33] Simo, J.C., Taylor, R.L. and Pister, K.S. Variational and projection methods for the volume constraint in finite deformation elasto-plasticity, Comp. Meth. in Appl. Mech. and Eng. (1985) $51,177-208$. 
[34] Simo, J.C. and Rifai, M.S. A class of mixed assumed strain methods and the method of incompatible modes, Int. Jour. for Num. Meths. in Eng. (1990) 29, 1595-1638.

[35] de Souza Neto, E.A., Pires, F.M.A. and Owen D.R.J. F-bar-based linear triangles and tetrahedra for finite strain analysis of nearly incompressible solids. Part I: formulation and benchmarking.Int. Jour. for Num. Meths. in Eng. (2005) 62, 353-383. 